\title{
On the viscoelastic equation with Balakrishnan- Taylor damping and nonlinear boundary/interior sources with variable-exponent nonlinearities
}

\author{
Abita Rahmoune and Benyattou Benabderrahmane
}

\begin{abstract}
This work is devoted to the study of a nonlinear viscoelastic Kirchhoff equation with Balakrishnan-Taylor damping and nonlinear boundary interior sources with variable exponents. Under appropriate assumptions, we establish a uniform decay rate of the solution energy in terms of the behavior of the nonlinear feedback and the relaxation function, without setting any restrictive growth assumptions on the damping at the origin and weakening the usual assumptions on the relaxation function.
\end{abstract}

Mathematics Subject Classification (2010): 49Q15, 35L05, 35L20 35B40, 35B35.

Keywords: Balakrishnan-Taylor damping, global existence, general decay, relaxation function, viscoelastic equation, Lebesgue and Sobolev spaces with variable exponents.

\section{Introduction}

In this paper, we study the following viscoelastic problem with BalakrishnanTaylor damping and nonlinear boundary interior sources involving the variableexponent nonlinearities

$$
\begin{gathered}
\frac{\partial^{2} u}{\partial t^{2}}-M\left(|\nabla u(t)|^{2}\right) \Delta u+\int_{0}^{t} g(t-s) \Delta u(s) \mathrm{d} s=|u|^{p(x)-1} u \text { in } \Omega \times(0,+\infty), \\
u=0 \text { on } \Gamma_{0} \times(0,+\infty), \\
M\left(|\nabla u(t)|^{2}\right) \frac{\partial u}{\partial \nu}-\int_{0}^{t} g(t-s) \frac{\partial}{\partial \nu} u(s) \mathrm{d} s+h\left(u_{t}\right)=|u|^{k(x)-1} u \text { on } \Gamma_{1} \times(0,+\infty), \\
u(x, 0)=u_{0}(x), u_{t}(x, 0)=u_{1}(x), x \in \Omega,
\end{gathered}
$$


where $M(r)$ is a locally Lipschitz function in $r, g>0$ is a memory kernel and $\Omega \subset \mathbb{R}^{n}$ $(n \geq 2)$, be a bounded domain with a smooth boundary $\Gamma=\partial \Omega=\Gamma_{0} \cup \Gamma_{1}$. The boundary $\Gamma$ of $\Omega$ is assumed to be regular and is divided by two closed and disjoint parts $\Gamma_{0}, \Gamma_{1}$, Here, $\Gamma_{0} \neq \emptyset$. (.) $)^{\prime}$ denotes the derivative with respect to time $t$ thus $u_{t}=\frac{\partial u}{\partial t}, u_{t t}=\frac{\partial^{2} u}{\partial t^{2}}, \Delta$ stands for the Laplacian with respect to the spatial variables, respectively. Let $\nu$ be the outward normal to $\Gamma$. The exponents $k($.$) and p($.$) are given$ measurable functions on $\Omega$ satisfying

$$
\left\{\begin{array}{l}
1<p^{-} \leq p(x) \leq p^{+}<\infty \\
1<k^{-} \leq k(x) \leq k^{+}<\infty
\end{array}\right.
$$

where

$$
\begin{cases}p^{+}=e s s \sup _{x \in \Omega}(x), & p^{-}=\underset{x \in \Omega}{e s s \inf p}(x), \\ k^{+}=e s s \sup _{x \in \Omega}(x), & k^{-}=e s s \inf _{x \in \Omega} k(x) .\end{cases}
$$

We also assume that $k$ satisfies the following Zhikov-Fan uniform local continuity condition:

$$
|k(x)-k(y)| \leq \frac{M}{|\log | x-y||}, \text { for all } x, y \text { in } \Omega \text { with }|x-y|<\frac{1}{2}, M>0 .
$$

In recent years, many authors have paid attention to the study of nonlinear hyperbolic, parabolic and elliptic equations with nonstandard growth condition. For instance, modeling of physical phenomena such as flows of electro-rheological fluids or fluids with temperature-dependent viscosity, thermoelasticity, nonlinear viscoelasticity, filtration processes through a porous media and image processing. More details on these problems can be found in $[5,7,1,2,3,26,34,35]$ and references therein.

Constant exponent. In (1.1)-(1.4), when $g \geq 0$ and $k, p$ are constants, this equation has its origin in the nonlinear vibration of an elastic string, were the source term $|u|^{p-2} u$ forces the negative-energy solutions to explode in finite time. While, the dissipation term $h\left(u_{t}\right)$ assures the existence of global solutions for any initial data, local, global existence and long-time behavior have been considered by many authors (see for example [40, 31, 19, 41] and references therein). It is well known that Kirchhoff first investigated the following nonlinear vibration of an elastic string for $f=g=0$ :

$$
\rho h \frac{\partial^{2} u}{\partial t^{2}}+\delta \frac{\partial u}{\partial t}+g\left(\frac{\partial u}{\partial t}\right)=\left\{p_{0}+\frac{E h}{2 L} \int_{0}^{L}\left(\frac{\partial u}{\partial x}\right)^{2} \mathrm{~d} x\right\} \frac{\partial^{2} u}{\partial x^{2}}+f(u)
$$

for $0<x<L, t \geq 0$, where $u(x, t)$ is the lateral displacement, $E$ the Young modulus, $\rho$ the mass density, $h$ the cross-section area, $L$ the length, $p_{0}$ the initial axial tension, $\delta$ the resistance modulus, and $f$ and $g$ the external forces. The above equation is described by the second order hyperbolic equation (1.1) and it is seemed to be important and natural that the equation with external forces is considered for analyzing phenomena in real world. The equations in (1.1)-(1.4) with $M \equiv 1$ form a class of nonlinear viscoelastic equations used to investigate the motion of viscoelastic materials. As these materials have a wide application in the natural sciences, their dynamics are interesting and of great importance. Hence, questions related to the behavior of the solutions for the wave equation with Dirichlet's boundary condition has attracted 
considerable the attention of many authors. In particular, there are many results of proving the nonexistence and blow-up of solutions with negative initial energy (see $[24,25,22,38,32,9]$ and a list of references therein) also these results were obtained with convexity method. However much less is known when the initial energy is positive (cf. [8, 21, 33, 42]) and these results used several, for example, contradiction method, decomposition method and so on. The equations in (1.1) with $M(r)=a+b r$ and $a>0, b>0$ is the model to describe the motion of deformable solids as hereditary effect is incorporated, which was first studied by Torrejon and Yong [37]. They proved the existence of weakly asymptotic stable solution for the large analytical datum. Later, Rivera [30] showed the existence of global solutions for small datum and the total energy decays to zero exponentially under some restrictions. Problem (1.1)-(1.4) is the extension of the problems in which the variable-exponent are constants and $g \geq 0$. The main difficulty of this problem is related to the presence of the quasilinear terms in (1.1)-(1.4) in the variable-exponent. In this paper a class of a weakly damped wave equation of generalized Kirchhoff type with nonlinear damping and source terms involving the variable-exponent nonlinearities were considered. Hence by using the Faedo-Galerkin arguments and compactness method as in [27], together with the Banach fixed point theorem, we will show the local existence of the problem (1.1)-(1.4). The purpose of this paper is to generalize the existence and uniform decay theorems of local solutions due to the constant-exponents. In other words we prove the existence and uniform decay rate of local solutions to weakly damped degenerate wave equations of Kirchhoff type (1.1)-(1.4) with nonlinear damping and source terms. This paper consists of 3 sections in addition to the introduction. In Section 2 , we recall the definitions of the variable-exponent Lebesgue spaces $L^{p(.)}(\Omega)$, the Sobolev spaces $W^{1, p(.)}(\Omega)$, and some of their properties. In Section 3, we state, with the proof, existence and uniqueness result of weak solution for (1.1)-(1.4) by employing Faedo-Galerkin's together with the Banach fixed point theorem and compactness methods. In Section 4, the statement and the proof of our global existence and a stability theorem for certain solutions with positive initial energy. To the best of our knowledge, this problem has not been studied previously. In addition, our method of determining these results, because the presence of the exponents $m($.$) and p($.$) , is$ somewhat different.

\section{Preliminaries. Function spaces}

In this section, we list and recall some well-known results and facts from the theory of the Sobolev spaces with variable exponent. (On the basic properties of the spaces $W^{1, p(x)}(\Omega)$ and $L^{p(x)}(\Gamma)$ we refer to $\left.[10,11,15,17,23]\right)$.

Throughout the rest of the paper we assume that $\Omega$ is a bounded domain of $\mathbb{R}^{n}$, $n \geq 1$ with smooth boundary $\Gamma$ and assume that $p($.$) is a measurable function on \bar{\Omega}$ such that:

$$
1<p^{-} \leq p(x) \leq p^{+}<\infty
$$

where

$$
p^{+}=e s s \sup _{x \in \Omega}(x), \quad p^{-}=e s s \inf _{x \in \Omega} p(x) .
$$


We also assume that $p$ satisfies the following Zhikov-Fan uniform local continuity condition:

$$
|p(x)-p(y)| \leq \frac{M}{|\log | x-y||}, \text { for all } x, y \text { in } \Omega \text { with }|x-y|<\frac{1}{2}, M>0 .
$$

Given a function $p: \bar{\Omega} \rightarrow\left[p^{-}, p^{+}\right] \subset(1, \infty), p^{ \pm}=$const, we define the set

$$
L^{p(.)}(\Omega)=\left\{\begin{array}{c}
v: \Omega \rightarrow \mathbb{R}: v \text { measurable functions on } \Omega, \\
\varrho_{p(.), \Omega}(v)=\int_{\Omega}|v(x)|^{p(x)} \mathrm{d} x<\infty .
\end{array}\right\}
$$

The variable-exponent space $L^{p(\cdot)}(\Omega)$ equipped with the Luxemburg norm

$$
\|u\|_{p(.), \Omega}=\|u\|_{p(.)}=\inf \left\{\lambda>0, \int_{\Omega}\left|\frac{u}{\lambda}\right|^{p(x)} \mathrm{d} x \leq 1\right\},
$$

becomes a Banach space.

In general, variable-exponent Lebesgue spaces are similar to classical Lebesgue spaces in many aspects, see the first discussed the $L^{p(x)}$ spaces and $W^{k, p(x)}$ spaces by Kovàcik and Rákosnik in [23].

Let us list some properties of the spaces $L^{p(.)}(\Omega)$ which will be used in the study of the problem (1.1)-(1.4).

- If $p(x)$ is measurable and $1<p^{-} \leq p(x) \leq p^{+}<\infty$ in $\Omega$, then $L^{p(.)}(\Omega)$ is a reflexive and separable Banach space and $C_{0}^{\infty}(\Omega)$ is dense in $L^{p(.)}(\Omega)$.

- If condition (2.2) is fulfilled, and $\Omega$ has a finite measure and $p, q$ are variable exponents so that $p(x) \leq q(x)$ almost everywhere in $\Omega$, the inclusion $L^{q(\cdot)}(\Omega) \subset$ $L^{p(\cdot)}(\Omega)$ is continuous and

$$
\forall v \in L^{q(.)}(\Omega) \quad\|u\|_{p(.)} \leq C\|u\|_{q(.)} ; \quad C=C\left(|\Omega|, p^{ \pm}\right)
$$

- The variable Sobolev space $W^{1, p(.)}(\Omega)$ is defined as the closure of $C_{0}^{\infty}(\Omega)$ with respect to the norm

$$
\|u\|_{W_{0}^{1, p(.)}(\Omega)}=\|u\|_{p(.), \Omega}+\|\nabla u\|_{p(.), \Omega} \cdot
$$

It is known that for the elements of $W_{0}^{1, p(.)}(\Omega)$ the Poincar'e inequality holds,

$$
\|u\|_{p(.), \Omega} \leq C(n, \Omega)\|\nabla u\|_{p(.), \Omega},
$$

and an equivalent norm of $W_{0}^{1, p(.)}(\Omega)$ can be defined by

$$
\|u\|_{W_{0}^{1, p(.)}(\Omega)}=\|\nabla u\|_{p(.), \Omega} \cdot
$$

According to $(2.2) W_{0}^{1, p(.)}(\Omega) \subset W_{0}^{1, p^{-}}(\Omega)$. If $p^{-}>\frac{2 n}{n+2}$, then the embedding $W_{0}^{1, p^{-}}(\Omega) \subset L^{2}(\Omega)$ is compact.

- It is known that $C_{0}^{\infty}(\Omega)$ is dense in $W_{0}^{1, p(.)}(\Omega)$ according to $(2.2)$ if $p(x) \in$ $C_{\log }(\bar{\Omega})$, that is, the variable exponent $p(x)$ is continuous in $\bar{\Omega}$ with the logarithmic module of continuity.

- It follows directly from the definition of the norm that

$$
\min \left(\|u\|_{p(.)}^{p^{-}},\|u\|_{p(.)}^{p^{+}}\right) \leq \varrho_{p(.)}(u) \leq \max \left(\|u\|_{p(.)}^{p^{-}},\|u\|_{p(.)}^{p^{+}}\right) .
$$


- The following generalized Hölder inequality

$$
\int_{\Omega}|u(x) v(x)| \mathrm{d} x \leq\left(\frac{1}{p^{-}}+\frac{1}{\left(p^{-}\right)^{\prime}}\right)\|u\|_{p(x)}\|v\|_{p^{\prime}(x)} \leq 2\|u\|_{p(x)}\|v\|_{p^{\prime}(x)}
$$

holds, for all $u \in L^{p(.)}(\Omega), v \in L^{p^{\prime}(.)}(\Omega)$ with $p(x) \in(1, \infty), p^{\prime}(x)=\frac{p(x)}{p(x)-1}$.

- If $p: \Omega \rightarrow\left[p^{-}, p^{+}\right] \subset[1,+\infty)$ is a measurable function and $p_{*}>\operatorname{ess} \sup p(x)$ with $p_{*} \leq \frac{2 n}{n-2}$, then the embedding $H_{0}^{1}(\Omega)=W_{0}^{1,2}(\Omega) \hookrightarrow L^{p(.)}(\Omega)$ is continuous and compact.

Lemma 2.1. ([10]) Let $\Omega$ be a bounded domain of $\mathbb{R}^{n}, p($.$) and m($.$) satisfies (1.5) and$ (2.2), then

$$
B_{0}\|\nabla u\|_{m(.)} \geq\|u\|_{p(.)} \quad \text { for all } u \in W_{0}^{1, m(.)}(\Omega) .
$$

where the optimal constant of Sobolev embedding $B_{0}$ is depends on $p^{ \pm}$and $|\Omega|$.

Lemma 2.2 (Poincaré's Inequality). ([10]) Let $\Omega$ be a bounded domain of $\mathbb{R}^{n}$ and $p($. satisfies (2.2), then

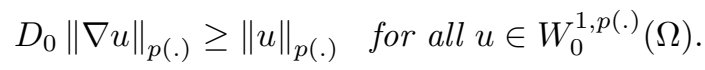

where the optimal constant of Sobolev embedding $D_{0}$ is depends on $p^{ \pm}$and $|\Omega|$.

Proposition 2.3. (See $[16,14,15,12,13])$ Let $\Omega$ be a bounded domain in $\mathbb{R}^{n}, p \in$ $C^{0,1}(\bar{\Omega}), 1<p^{-} \leq p(x) \leq p^{+}<n$. Then For any $q \in C(\Gamma)$ with $1 \leq q(x) \leq$ $\frac{(n-1) p(x)}{n-p(x)}$, there is a continuous trace $W^{1, p(x)}(\Omega) \rightarrow L^{q(x)}(\Gamma)$, when $1 \leq q(x)<<$ $\frac{(n-1) p(x)}{n-p(x)}$, the trace is compact, in particulary the continuous trace $W^{1, p(x)}(\Omega) \rightarrow$ $L^{p(x)}(\Gamma)$ is compact.

There are many proprieties of the theory of Lebesgue and Sobolev spaces with variable exponent, see the detailed exposition given in the monograph [4, Ch.1.].

Lemma 2.4 (Modified Gronwall inequality). Let $\phi$ and $h$ be nonnegative functions on $[0,+\infty)$ satisfying

$$
0 \leq \phi \leq K+\int_{0}^{t} h(s) \phi(s)^{r+1} \mathrm{~d} s,
$$

with $K>0$ and $r>0$. Then

$$
\phi(t) \leq\left(K^{-r}-r \int_{0}^{t} h(s) \mathrm{d} s\right)^{\frac{-1}{r}} .
$$

as long as the right-hand side exists. 


\subsection{Mathematical hypotheses}

We begin this section by introducing some hypotheses and our main result. Throughout this paper, we use standard functional spaces and denote that $\|\cdot\|_{p(.)}$, $\|\cdot\|_{p(.), \Gamma_{1}}$ are $L^{p(.)}(\Omega)$ norm and $L^{p(.)}\left(\Gamma_{1}\right)$ norm, respectively, such that:

$$
\begin{gathered}
\|u\|_{p(.), \Gamma_{1}}=\int_{\Gamma_{1}}|u(\eta)|^{p(\eta)} \mathrm{d} \eta=\int_{\Gamma_{1}}|u(x)|^{p(x)} \mathrm{d} \Gamma ; \\
\|\cdot\|_{q, \Gamma_{1}}=\int_{\Gamma_{1}}|u(x)|^{q} \mathrm{~d} \Gamma .
\end{gathered}
$$

Also, we define $(u, v)=\int_{\Omega} u(x) v(x) \mathrm{d} x$ and $(u, v)_{\Gamma_{1}}=\int_{\Gamma_{1}} u(x) v(x) \mathrm{d} \Gamma$.

The inner products and norms in $L^{2}(\Omega)$ and $H_{0}^{1}(\Omega)$ are represented by $(.,),.\|$. respectively and they are given by:

$$
\begin{gathered}
(u, v)=\int_{\Omega} u(x) v(x) \mathrm{d} x \text { and }\|u\|_{L^{2}(\Omega)}^{2}=|u|^{2}=\int_{\Omega} u^{2} \mathrm{~d} x \\
\|u\|_{H_{0}^{1}(\Omega)}^{2}=\|u\|^{2}=|\nabla u|^{2}=\int_{\Omega}|\nabla u|^{2} \mathrm{~d} x .
\end{gathered}
$$

Next, we state the assumptions for problem (1.1)-(1.4).

(H1) Hypotheses on $M$. Let $M \in C\left([0,+\infty), \mathbb{R}_{+}\right)$be a nonnegative locally Lipschitz function and for positive constant $m>0$, we have

$$
M(s) \geq m_{3}>0, s \geq 0
$$

(H2) Hypotheses on $g \cdot g:[0, \infty) \rightarrow(0, \infty)$ is a bounded $C^{1}$ function satisfying

$$
g(0)>0, m_{3}-\int_{0}^{\infty} g(s) \mathrm{d} s=l>0,
$$

and there exists a non-increasing positive differentiable function $\zeta$ such that

$$
g^{\prime}(t) \leq-\zeta(t) g(t) \text { for all } t \geq 0 .
$$

(H3) Hypotheses on $h . h: \mathbb{R} \rightarrow \mathbb{R}$ is a Lipschitz non-decreasing function with

$$
h(s) s \geq 0 \text { for all } s \in \mathbb{R}
$$

(H4) Hypotheses on $p(),. k($.$) . Let m($.$) and p($.$) are given measurable functions on$ $\bar{\Omega}$ satisfying the following conditions,

$1<p^{-} \leq p(x) \leq p^{+} \leq \frac{n}{n-2}, n>2$ and $1 \leq p^{-} \leq p^{+}<\infty$ if $n=2$,

$1<k^{-} \leq k(x) \leq k^{+}<\frac{n-1}{n-2}, n>2$ and $1 \leq k^{-} \leq k^{+}<\infty$ if $n=2$

According to (2.12), we have

$$
\|u\|_{p^{+}+1} \leq B|\nabla u| \quad \forall u \in H_{\Gamma 0}^{1}(\Omega) .
$$

where

$$
H_{\Gamma_{0}}^{1}(\Omega)=\left\{u \in H^{1}(\Omega):\left.u\right|_{\Gamma_{0}}=0\right\}
$$

endow with the Hilbert structure induced by $H^{1}(\Omega)$, is a Hilbert space and $B>0$ be the optimal constant of Sobolev embedding $H_{\Gamma_{0}}^{1}(\Omega) \hookrightarrow L^{p^{+}+1}(\Omega)$ which satisfies the inequality (2.13) and we use the trace-Sobolev imbedding: 
$H_{\Gamma_{0}}^{1}(\Omega) \hookrightarrow L^{k^{+}+1}\left(\Gamma_{1}\right), 1<k^{+}<\frac{n-1}{n-2}$. In this case, the imbedding constant is denoted by $B_{*}$, i.e.,

$$
\|u\|_{k^{+}+1, \Gamma_{1}} \leq B_{*}|\nabla u| \quad \forall u \in H_{\Gamma_{0}}^{1}(\Omega) .
$$

(H5) Assumptions on $u_{0}, u_{1}$. Assume that $\left(u_{0}, u_{1}\right) \in H_{\Gamma_{0}}^{1}(\Omega) \cap H^{2}(\Omega) \times H_{\Gamma_{0}}^{1}(\Omega)$ satisfying the compatibility conditions

$$
M\left(\left|\nabla u_{0}\right|^{2}\right) \frac{\partial u_{0}}{\partial \nu}+h\left(u_{1}\right)=\left|u_{0}\right|^{k(.)-1} u_{0} \text { on } \Gamma_{1} .
$$

\section{Main result}

This section first presents the local existence and uniqueness of the solution for problem (1.1)-(1.4) with a degenerated second order equation on $\Gamma_{1}$. Our method of proof by perturbing the boundary equation is based on the combination of the FaedoGalerkin approximation and the compactness method together with the Banach fixed point theorem with the ones from [36].

\subsection{Existence of local solutions}

In this section, under the assumptions $\left(H_{1}\right)-\left(H_{5}\right)$, we prove the existence of the local solution to the wave equation of Kirchhoff type (1.1)-(1.4) for any initial value $\left(u_{0}, u_{1}\right) \in H_{\Gamma_{0}}^{1}(\Omega) \cap H^{2}(\Omega) \times H_{\Gamma_{0}}^{1}(\Omega)$. First we need the local existence and uniqueness of the solution to the following wave equation:

$$
\begin{gathered}
\frac{\partial^{2} u}{\partial t^{2}}-M\left(|\nabla \varphi(t)|^{2}\right) \Delta u+\int_{0}^{t} g(t-s) \Delta u(s) \mathrm{d} s=|u|^{p(x)-1} u \text { in } \Omega \times(0,+\infty), \\
u=0 \text { on } \Gamma_{0} \times(0,+\infty), \\
M\left(|\nabla \varphi(t)|^{2}\right) \frac{\partial u}{\partial \nu}-\int_{0}^{t} g(t-s) \frac{\partial}{\partial \nu} u(s) \mathrm{d} s+h\left(u_{t}\right)=|u|^{k(x)-1} u \text { on } \Gamma_{1} \times(0,+\infty), \\
u(x, 0)=u_{0}(x), u_{t}(x, 0)=u_{1}(x), x \in \Omega,
\end{gathered}
$$

where $\varphi:[0, T] \rightarrow H_{\Gamma_{0}}^{1}(\Omega)$ is a continuous function. So we first prove the existence and uniqueness of the local solution to $(\mathrm{P} 4)$. Let $\left(w_{j}\right), j=1,2, \ldots$, be a completely orthonormal system in $L^{2}(\Omega)$ having the following properties:

$* \forall j ; w_{j} \in H_{\Gamma_{0}}^{1}(\Omega)$;

* The family $\left\{w_{1}, w_{2}, \ldots, w_{m}\right\}$ is linearly independent;

$* V_{m}$ the space generated by $\left\{w_{1}, w_{2}, \ldots, w_{m}\right\}, \underset{m}{\cup} V_{m}$ is dense in $H_{\Gamma_{0}}^{1}(\Omega) \cap H^{2}(\Omega)$. We construct approximate solutions, for each $\eta \in(0,1), u^{\eta m}(m=1,2,3, \ldots)$ in $V_{m}$ in the form:

$$
u^{\eta m}(t)=\sum_{i=1}^{m} K_{j m}(t) w_{i}, \quad m=1,2, \ldots
$$


where $K_{j m}(t)$ are determined by the following ordinary differential perturbed equation:

$$
\begin{gathered}
\left(u_{t t}^{\eta m}(t), w_{j}\right)+M\left(|\nabla \varphi(t)|^{2}\right)\left(\nabla u^{\eta m}, \nabla w_{j}\right)-\left(\int_{0}^{t} g(t-s) \nabla u^{\eta m}(s) \mathrm{d} s, \nabla w_{j}\right) \\
+\left(h\left(u_{t}^{\eta m}\right), w_{j}\right)_{\Gamma_{1}}+\eta\left(u_{t}^{\eta m}(t), w_{j}\right)_{\Gamma_{1}} \\
=\left(\left|u^{\eta m}(t)\right|^{k(x)-1} u^{\eta m}(t), w_{j}\right)_{\Gamma_{1}}+\left(\left|u^{\eta m}(t)\right|^{p(x)-1} u^{\eta m}(t), w_{j}\right), j=1,2, \ldots,
\end{gathered}
$$

and will be completed by the following initial conditions $u^{\eta m}(0), u_{t}^{\eta m}(0)$ which satisfies:

$$
\left\{\begin{array}{c}
u^{\eta m}(0)=u_{0}^{\eta m}=\sum_{i=1}^{m} \alpha_{i m} w_{i} \longrightarrow u_{0}(x) \text { when } m \longrightarrow \infty \text { in } H_{\Gamma_{0}}^{1}(\Omega) \cap H^{2}(\Omega), \\
u_{t}^{\eta m}(0)=u_{1}^{\eta m}=\sum_{i=1}^{m} \beta_{i m} w_{i} \longrightarrow u_{1}(x) \text { when } m \longrightarrow \infty \text { in } H_{\Gamma_{0}}^{1}(\Omega) .
\end{array}\right.
$$

Then it holds that for any given $v \in \operatorname{Span}\left\{w_{1}, w_{2}, \ldots, w_{m}\right\}$,

$$
\begin{gathered}
\left(u_{t t}^{\eta m}(t), v\right)+M\left(|\nabla \varphi(t)|^{2}\right)\left(\nabla u^{\eta m}, \nabla v\right)-\left(\int_{0}^{t} g(t-s) \nabla u^{\eta m}(s) \mathrm{d} s, \nabla v\right) \\
+\left(h\left(u_{t}^{\eta m}\right), v\right)_{\Gamma_{1}}+\eta\left(u_{t}^{\eta m}(t), v\right)_{\Gamma_{1}} \\
=\left(\left|u^{\eta m}(t)\right|^{k(x)-1} u^{\eta m}(t), v\right)_{\Gamma_{1}}+\left(\left|u^{\eta m}(t)\right|^{p(x)-1} u^{\eta m}(t), v\right) .
\end{gathered}
$$

By virtue of the theory of ordinary differential equations, system (3.1), (3.2) and (3.3) has a unique local solution which is extended to a maximal intervals $\left[0, t_{m}[\right.$.

A solution $u$ to the problem (1.1)-(1.4) on some interval $\left[0, t_{m}\right.$ [ will be obtain as the limit of $u^{\eta m}$ as $m \rightarrow \infty$ and $\eta \rightarrow 0$. Then, this solution can be extended to the whole interval $[0, T]$, for all $T>0$, as a consequence of the a priori estimates that shall be proven in the next step. In this paper, $\varepsilon, C(\varepsilon), C_{\varepsilon}, C, C\left(m_{3}\right), c, c^{*}$ or $c_{*}$ denote a various positive constant which changes from line to line and are independent of natural number $n$ and depends only (possibly) on the initial value.

Let us first recall a useful identity for the memory term who play an important role in the sequel. By denoting

$$
(g \diamond \nabla(u))(t)=\int_{0}^{t} g(t-s) \int_{\Omega}|\nabla u(s)-\nabla u(t)|^{2} \mathrm{~d} x \mathrm{~d} s,
$$

it is easy, by differentiating the term $(\beta \diamond \nabla(u))(t)$ with respect to $t$, to show that

$$
\begin{gathered}
\int_{\Omega} \int_{0}^{t} g(t-s) \nabla u(s) \nabla u_{t}(t) \mathrm{d} x \mathrm{~d} s \\
=-\frac{1}{2} \frac{\mathrm{d}}{\mathrm{d} t}\left\{(g \diamond \nabla u)(t)-|\nabla(u(t))|^{2} \int_{0}^{t} g(s) \mathrm{d} s\right\} \\
+\frac{1}{2}\left(g^{\prime} \diamond \nabla u\right)(t)-\frac{1}{2} g(t)|\nabla u(t)|^{2} .
\end{gathered}
$$

We prove by the Galerkin method the following lemma on the existence and uniqueness of local solution to $(\mathrm{P} 4)$ in time. 
Lemma 3.1. Let $M(r)$ be a nonnegative locally Lipschitz function. Let

$$
\left(u_{0}, u_{1}\right) \in H_{\Gamma_{0}}^{1}(\Omega) \cap H^{2}(\Omega) \times H_{\Gamma_{0}}^{1}(\Omega) .
$$

Assume that the differentiable function $\varphi(t)$ satisfies

$$
\varphi(0)=u_{0}, \varphi^{\prime}(0)=u_{1}
$$

Assume that the following condition is satisfied

$$
\begin{gathered}
1<k^{+}<\frac{n-1}{n-2} \text { and } 1<p^{+} \leq \frac{n}{n-2} \text { if } n \geq 3, \\
1 \leq k^{-} \leq k^{+}<\infty \text { and } 1 \leq p^{-} \leq p^{+}<\infty \text { if } n=2 .
\end{gathered}
$$

Then there exists a time $T_{0}=T_{0}\left(u_{0}, u_{1}, m_{1}, m_{2}, m_{3}\right)>0$ such that if there exist $m_{1}, m_{2}, m_{3}>0$ and $T>0$ satisfying

$$
|\nabla \varphi(t)| \leq m_{1},\left|\nabla \varphi^{\prime}(t)\right| \leq m_{2}, M\left(|\nabla \varphi(t)|^{2}\right) \geq m_{3}>0
$$

for all $t \in[0, T]$, then there exists a unique local weak solution in time $u(t)$ to $(\mathrm{P} 4)$ with the initial value $\left(u_{0}, u_{1}\right)$ on $\left[0, T_{0}\right]$, where $T_{0} \leq T$ satisfying:

$$
\begin{gathered}
\left.u(t) \in C\left(\left[0, T_{0}\right]: H_{\Gamma_{0}}^{1}(\Omega)\right)\right), \\
u_{t}(t) \in C\left(\left[0, T_{0}\right]: L^{2}(\Omega)\right) \cap C\left([0, T]: H_{\Gamma_{0}}^{1}(\Omega)\right), \\
u_{t t}(t) \in C\left(\left[0, T_{0}\right]: L^{2}(\Omega)\right) .
\end{gathered}
$$

Proof. The first estimate (Estimates on $u_{t}^{\eta m}$ ):

By taking $v=u_{t}^{\eta m}(t)$ in (3.3), we have that

$$
\begin{gathered}
\left(u_{t t}^{\eta m}(t), u_{t}^{\eta m}\right)+M\left(|\nabla \varphi(t)|^{2}\right)\left(\nabla u^{\eta m}, \nabla u_{t}^{\eta m}\right)-\left(\int_{0}^{t} g(t-s) \nabla u^{\eta m}(s) \mathrm{d} s, \nabla u_{t}^{\eta m}\right) \\
+\eta\left\|u_{t}^{\eta m}(t)\right\|_{2, \Gamma_{1}}^{2}+\left(h\left(u_{t}^{\eta m}\right), u_{t}^{\eta m}\right)_{\Gamma_{1}}=\left(\left|u^{\eta m}(t)\right|^{k(x)-1} u^{\eta m}(t), u_{t}^{\eta m}\right)_{\Gamma_{1}} \\
+\left(\left|u^{\eta m}(t)\right|^{p(x)-1} u^{\eta m}(t), u_{t}^{\eta m}\right) .
\end{gathered}
$$

Since it holds that

$$
\begin{gathered}
\int_{0}^{t} M\left(|\nabla \varphi(t)|^{2}\right)\left(\nabla u^{\eta m}, \nabla u_{t}^{\eta m}\right) \mathrm{d} s=\frac{1}{2} \int_{0}^{t} M\left(|\nabla \varphi(s)|^{2}\right) \frac{\mathrm{d}}{\mathrm{d} t}\left|\nabla u^{\eta m}(s)\right|^{2} \mathrm{~d} s \\
\geq\left[\frac{1}{2} M\left(|\nabla \varphi(s)|^{2}\right)\left|\nabla u^{\eta m}\right|^{2}\right]_{0}^{t}-\frac{1}{2} \int_{0}^{t}\left[\frac{\mathrm{d}^{+}}{\mathrm{d} s} M\left(|\nabla \varphi(s)|^{2}\right)\right]\left|\nabla u^{\eta m}\right|^{2} \mathrm{~d} s, \\
\frac{\mathrm{d}^{+}}{\mathrm{d} s} M\left(|\nabla \varphi(s)|^{2}\right) \leq 2\left(\frac{\mathrm{d}^{+}}{\mathrm{d} r} M(r)\right)|\nabla \varphi(s)|\left|\nabla \varphi^{\prime}(s)\right| \leq 2 L m_{1} m_{2}, \quad s \in\left[0, T_{1}\right] .
\end{gathered}
$$


where $L=L\left(m_{1}\right)$ is a local Lipschitz constant for $M(r)$, we have for $t \in\left(0, t_{m}\right)$

$$
\begin{aligned}
& \frac{1}{2}\left|u_{t}^{\eta m}\right|^{2}+ \frac{1}{2}\left(M\left(|\nabla \varphi(t)|^{2}\right)-\int_{0}^{t} g(s) \mathrm{d} s\right)\left|\nabla u^{\eta m}\right|^{2}+\left(g \diamond \nabla u^{\eta m}\right)(t) \\
&-\frac{1}{2} \int_{0}^{t}\left(g^{\prime} \diamond \nabla u^{\eta m}\right)(s) \mathrm{d} s+\left.\eta \int_{0}^{t}|| u_{t}^{\eta m}(s)\right|_{2, \Gamma_{1}} ^{2} \mathrm{~d} s \\
&+\frac{1}{2} \int_{0}^{t} g(s)\left|\nabla u^{\eta m}\right|^{2} \mathrm{~d} s+\int_{0}^{t}\left(h\left(u_{t}^{\eta m}\right), u_{t}^{\eta m}(s)\right)_{\Gamma_{1}} \mathrm{~d} s \\
& \leq L m_{1} m_{2} \int_{0}^{t}\left|\nabla u^{\eta m}\right|^{2} \mathrm{~d} s+\frac{1}{2} M\left(|\nabla \varphi(0)|^{2}\right)\left|\nabla u_{0}\right|^{2}+\frac{1}{2}\left|u_{1}\right|^{2} \\
&=\int_{0}^{t}\left(\left|u^{\eta m}(s)\right|^{k(x)-1} u^{\eta m}(s), u_{t}^{\eta m}\right)_{\Gamma_{1}} \mathrm{~d} s \\
&+\int_{0}^{t}\left(\left|u^{\eta m}(s)\right|^{p(x)-1} u^{\eta m}(s), u_{t}^{\eta m}\right) \mathrm{d} s .
\end{aligned}
$$

Young's inequality gives

$$
\begin{gathered}
\left.\left.\left|\int_{\Omega}\right| u^{\eta m}(t)\right|^{p(x)-1} u^{\eta m}(t) u_{t}^{\eta m}(t) \mathrm{d} x\left|\leq \int_{\Omega}\right| u^{\eta m}(t)\right|^{p(x)-1}\left|u^{\eta m}(t)\right|\left|u_{t}^{\eta m}(t)\right| \mathrm{d} x \\
\leq\left|u^{\eta m}(t)\right|^{p(x)-1}\left|u^{\eta m}(t)\right|\left|u_{t}^{\eta m}(t)\right| \\
\leq \frac{1}{2} C_{\varepsilon} \max \left(\int_{\Omega}\left|u^{\eta m}(t)\right|^{2 p^{+}} \mathrm{d} x, \int_{\Omega}\left|u^{\eta m}(t)\right|^{2 p^{-}} \mathrm{d} x\right)+\frac{1}{2} \varepsilon \int_{\Omega}\left|u_{t}^{\eta m}(t)\right|^{2} \mathrm{~d} x \\
\leq \frac{1}{2} C_{\varepsilon}\left(\left|\nabla u^{\eta m}\right|^{2 p^{+}}+\left|\nabla u^{\eta m}\right|^{2 p^{-}}\right)+\frac{1}{2} \varepsilon\left|u_{t}^{\eta m}(t)\right|^{2}
\end{gathered}
$$

Also

$$
\begin{gathered}
\left.\left|\int_{\Gamma_{1}}\right| u^{\eta m}(t)\right|^{k(x)-1} u^{\eta m}(t) u_{t}^{\eta m}(t) \mathrm{d} \Gamma \mid \\
\leq \frac{1}{2} C_{\varepsilon} \max \left(\left\|u^{\eta m}\right\|_{2 k^{+}, \Gamma_{1}}^{2 k^{+}},\left\|u^{\eta m}\right\|_{2 k^{-}, \Gamma_{1}}^{2 k^{-}}\right)+\frac{1}{2} \varepsilon \int_{\Gamma_{1}}\left|u_{t}^{\eta m}(t)\right|^{2} \mathrm{~d} \Gamma \\
\frac{1}{2} C_{\varepsilon}\left(\left|\nabla u^{\eta m}\right|^{2 k^{+}}+\left|\nabla u^{\eta m}\right|^{2 k^{-}}\right)+\frac{1}{2} \varepsilon\left\|u_{t}^{\eta m}(t)\right\|_{2, \Gamma_{1}}^{2},
\end{gathered}
$$

consequently, taking (2.8) and (2.9) into account

$$
\left(M\left(|\nabla \varphi(t)|^{2}\right)-\int_{0}^{t} g(s) \mathrm{d} s\right) \geq m_{3}-\int_{0}^{\infty} g(s) \mathrm{d} s=l>0
$$


Combining above results, and observing that $g>0$ and $g^{\prime} \leq 0$, we deduce

$$
\begin{gathered}
\frac{1}{2}\left|u_{t}^{\eta m}\right|^{2}+\frac{1}{2} l\left|\nabla u^{\eta m}\right|^{2}+\left(g \diamond \nabla u^{\eta m}\right)-\frac{1}{2} \int_{0}^{t}\left(g^{\prime} \diamond \nabla u^{\eta m}\right)(s) \mathrm{d} s \\
+\eta \int_{0}^{t}|| u_{t}^{\eta m}(s) \|_{2, \Gamma_{1}}^{2} \mathrm{~d} s+\frac{1}{2} \int_{0}^{t} g(s)\left|\nabla u^{\eta m}\right|^{2} \mathrm{~d} s+\int_{0}^{t}\left(h\left(u_{t}^{\eta m}\right), u_{t}^{\eta m}(s)\right)_{\Gamma_{1}} \mathrm{~d} s \\
\leq L m_{1} m_{2} \int_{0}^{t}\left|\nabla u^{\eta m}\right|^{2} \mathrm{~d} s+\frac{1}{2} M\left(|\nabla \varphi(0)|^{2}\right)\left|\nabla u_{0}\right|^{2}+\frac{1}{2}\left|u_{1}\right|^{2} \\
+C_{\varepsilon} \int_{0}^{t}\left(\left|\nabla u^{\eta m}\right|^{2 k^{+}}+\left|\nabla u^{\eta m}\right|^{2 k^{-}}+\left|\nabla u^{\eta m}\right|^{2 p^{+}}+\left|\nabla u^{\eta m}\right|^{2 p^{-}}\right) \mathrm{d} s \\
+\frac{1}{2} \varepsilon \int_{0}^{t}\left|u_{t}^{\eta m}(s)\right|^{2} \mathrm{~d} s+\varepsilon \int_{0}^{t}|| u_{t}^{\eta m}(s) \|_{2, \Gamma_{1}}^{2} \mathrm{~d} s,
\end{gathered}
$$

Choosing $\varepsilon=\frac{\eta}{2}$, we arrive at

$$
\begin{gathered}
\frac{1}{2}\left|u_{t}^{\eta m}\right|^{2}+\frac{1}{2} l\left|\nabla u^{\eta m}\right|^{2}+\left(g \diamond \nabla u^{\eta m}\right)(t)-\frac{1}{2} \int_{0}^{t}\left(g^{\prime} \diamond \nabla u^{\eta m}\right)(s) \mathrm{d} s \\
+\left.\frac{\eta}{2} \int_{0}^{t}|| u_{t}^{\eta m}(s)\right|_{2, \Gamma_{1}} ^{2} \mathrm{~d} s+\frac{1}{2} \int_{0}^{t} g(s)\left|\nabla u^{\eta m}(s)\right|^{2} \mathrm{~d} s+\int_{0}^{t}\left(h\left(u_{t}^{\eta m}\right), u_{t}^{\eta m}(s)\right)_{\Gamma_{1}} \mathrm{~d} s \\
\leq \frac{\eta}{2} \int_{0}^{t}\left|u_{t}^{\eta m}(s)\right|^{2} \mathrm{~d} s+L m_{1} m_{2} \int_{0}^{t}\left|\nabla u^{\eta m}\right|^{2} \mathrm{~d} s \\
+C_{\varepsilon} \int_{0}^{t}\left(\left|\nabla u^{\eta m}\right|^{2 k^{+}}+\left|\nabla u^{\eta m}\right|^{2 k^{-}}+\left|\nabla u^{\eta m}\right|^{2 p^{+}}+\left|\nabla u^{\eta m}\right|^{2 p^{-}}\right) \mathrm{d} s \\
+\frac{1}{2} L m_{1}\left|\nabla u_{0}\right|^{2}+\frac{1}{2}\left|u_{1}\right|^{2}+C_{\varepsilon} .
\end{gathered}
$$

Thus, there exist $B>0, \beta>0$ and $r>0$ such that

$$
\left|\nabla u^{\eta m}\right|^{2}+\left|u_{t}^{\eta m}\right|^{2} \leq B+\beta \int_{0}^{t}\left[1+\left(\left|\nabla u^{\eta m}(s)\right|^{2}+\left|u_{t}^{\eta m}(s)\right|^{2}\right)^{r+1}\right] \mathrm{d} s
$$

where we note that $B$ and $\beta$ are independent of $m$ and $r$. Since $r>0$, there exists an enough small time $T_{0}:=T_{0}\left(u_{0}, u_{1}, m_{3}\right) \in\left(0, T_{1}\right)$ satisfying

$$
\left(B+\beta T_{0}\right)^{-r}-r \beta T_{0}>0
$$

Thus, we have by the modified Gronwall lemma 2.4

$$
\left|\nabla u^{\eta m}\right|^{2}+\left|u_{t}^{\eta m}\right|^{2} \leq\left(\left(B+\beta T_{0}\right)^{-r}-r \beta T_{0}\right)^{\frac{-1}{r}}
$$

Therefore, there exist constants $c_{i}=c_{i}\left(u_{0}, u_{1}, m_{3}\right)>0(i=1,2,3)$ such that for any $t \in\left[0, T_{0}\right]$

$$
\left|\nabla u^{\eta m}\right|^{2} \leq C_{1} \text { and }\left|u_{t}^{\eta m}\right|^{2} \leq C_{2}
$$


Furthermore, by (3.8) it follows that

$$
\begin{aligned}
(g \diamond & \left.\nabla u^{\eta m}\right)(t)-\frac{1}{2} \int_{0}^{t}\left(g^{\prime} \diamond \nabla u^{\eta m}\right)(s) \mathrm{d} s+\int_{0}^{t}\left\|u_{t}^{\eta m}(s)\right\|_{2, \Gamma_{1}}^{2} \mathrm{~d} s \\
& +\int_{0}^{t} g(s)\left|\nabla u^{\eta m}(s)\right|^{2} \mathrm{~d} s+\int_{0}^{t}\left(h\left(u_{t}^{\eta m}\right), u_{t}^{\eta m}(s)\right)_{\Gamma_{1}} \mathrm{~d} s \leq C_{3}
\end{aligned}
$$

where $C_{i}$ are a positive constants which are independent of $m, \eta$ and $t$. Thus, the solution can be extended to $[0, T)$ and, in addition, we have

$$
\begin{gathered}
\left(u^{\eta m}\right) \text { is bounded sequence in } L^{\infty}\left(0, T ; H_{\Gamma_{0}}^{1}(\Omega)\right), \\
\left(u_{t}^{\eta m}\right) \text { is bounded sequence in } L^{\infty}\left(0, T ; L^{2}(\Omega)\right), \\
\left(h\left(u_{t}^{\eta m}\right) \cdot u_{t}^{\eta m}\right) \text { is bounded sequence in } L^{1}\left(0, T ; L^{1}\left(\Gamma_{1}\right)\right) .
\end{gathered}
$$

The second estimate (Estimates on $u_{t t}^{\eta m}$ ):

First of all, we are going to estimate $u_{t t}^{\eta m}(0)$. By taking $t=0$ in (3.3), taking (2.15) into account, we get

$$
\begin{aligned}
\left|u_{t t}^{\eta m}(0)\right|^{2} & \leq c\left|M\left(\left|\nabla u_{0}\right|^{2}\right)\right|^{2}\left|\Delta u_{0}\right|^{2}+c \int_{\Omega}\left|u_{0}\right|^{2 p(x)} \mathrm{d} x \\
& \leq c L\left|\nabla u_{0}\right|^{4}\left|\Delta u_{0}\right|^{2}+c \max \left(\left|\nabla u_{0}\right|^{2 p^{+}},\left|\nabla u_{0}\right|^{2 p^{-}}\right) \leq c^{*}
\end{aligned}
$$

Now, by differentiating (3.3) with respect to $t$ and substituting $w_{j}=u_{t t}^{\eta m}(t)$, we have

$$
\begin{gathered}
\frac{1}{2} \frac{\mathrm{d}}{\mathrm{d} t}\left|u_{t t}^{\eta m}\right|^{2}+2 M^{\prime}\left(|\nabla \varphi(t)|^{2}\right)\left(\nabla \varphi, \nabla \varphi^{\prime}\right)\left(\nabla u^{\eta m}, \nabla u_{t t}^{\eta m}\right) \\
+M\left(|\nabla \varphi(t)|^{2}\right)\left(\nabla u_{t}^{\eta m}, \nabla u_{t t}^{\eta m}\right) \\
+\left(h^{\prime}\left(u_{t}^{\eta m}\right) u_{t t}^{\eta m}, u_{t t}^{\eta m}\right)_{\Gamma_{1}}+\left.\eta|| u_{t t}^{\eta m}(s)\right|_{2, \Gamma_{1}} ^{2}=\left(k(x)\left|u^{\eta m}(t)\right|^{k(x)-1} u_{t}^{\eta m}(t), u_{t t}^{\eta m}\right)_{\Gamma_{1}} \\
+\left(p(x)\left|u^{\eta m}(t)\right|^{p(x)-1} u_{t}^{\eta m}(t), u_{t t}^{\eta m}\right)+g(0) \frac{\mathrm{d}}{\mathrm{d} t}\left(\nabla u^{\eta m}(t), \nabla u_{t}^{\eta m}\right)-g(0)\left|\nabla u_{t}^{\eta m}\right|^{2} \\
+\frac{\mathrm{d}}{\mathrm{d} t}\left(\int_{0}^{t} g^{\prime}(t-s) \nabla u^{\eta m}(s) \mathrm{d} s, \nabla u_{t}^{\eta m}\right)-g^{\prime}(0)\left(\nabla u^{\eta m}, \nabla u_{t}^{\eta m}(t)\right) \\
-\left(\int_{0}^{t} g^{\prime \prime}(t-s) \nabla u^{\eta m}(s) \mathrm{d} s, \nabla u_{t}^{\eta m}\right) .
\end{gathered}
$$

To analyze the term $2 M^{\prime}\left(|\nabla \varphi(t)|^{2}\right)\left(\nabla \varphi, \nabla \varphi^{\prime}\right)\left(\nabla u^{\eta m}, \nabla u_{t t}^{\eta m}(t)\right)$, we multiplying both sides of (3.3) by

$$
f(t)=\frac{2 M^{\prime}\left(|\nabla \varphi(t)|^{2}\right)\left(\nabla \varphi, \nabla \varphi^{\prime}\right)}{M\left(|\nabla \varphi(t)|^{2}\right)}\left(\leq \frac{2 L m_{1} m_{2}}{m_{3}}\right)
$$


and replacing $v=u_{t t}^{\eta m}(t)$, we have

$$
\begin{gathered}
2 M^{\prime}\left(|\nabla \varphi(t)|^{2}\right)\left(\nabla \varphi, \nabla \varphi^{\prime}\right)\left(\nabla u^{\eta m}, \nabla u_{t t}^{\eta m}\right)=-f(t)\left|u_{t t}^{\eta m}\right|^{2} \\
+f(t)\left(\int_{0}^{t} g(t-s) \nabla u^{\eta m}(s) \mathrm{d} s, \nabla u_{t t}^{\eta m}\right) \\
-f(t)\left(h\left(u_{t}^{\eta m}\right), u_{t t}^{\eta m}\right)_{\Gamma_{1}}-\eta f(t)\left(u_{t}^{\eta m}(t), u_{t t}^{\eta m}\right)_{\Gamma_{1}} \\
+f(t)\left(\left|u^{\eta m}(t)\right|^{k(x)-1} u^{\eta m}(t), u_{t t}^{\eta m}\right)_{\Gamma_{1}} \\
+f(t)\left(\left|u^{\eta m}(t)\right|^{p(x)-1} u^{\eta m}(t), u_{t t}^{\eta m}\right)
\end{gathered}
$$

By replacing above equality in (3.12), we have

$$
\begin{gathered}
\frac{1}{2} \frac{\mathrm{d}}{\mathrm{d} t}\left|u_{t t}^{\eta m}\right|^{2}+f(t)\left(\int_{0}^{t} g(t-s) \nabla u^{\eta m}(s) \mathrm{d} s, \nabla u_{t t}^{\eta m}\right) \\
+f(t)\left(\left|u^{\eta m}(t)\right|^{k(x)-1} u^{\eta m}(t), u_{t t}^{\eta m}\right)_{\Gamma_{1}} \\
+f(t)\left(\left|u^{\eta m}(t)\right|^{p(x)-1} u^{\eta m}(t), u_{t t}^{\eta m}\right) \\
+\eta \|\left. u_{t t}^{\eta m}(s)\right|_{2, \Gamma_{1}} ^{2}+M\left(|\nabla \varphi(t)|^{2}\right)\left(\nabla u_{t}^{\eta m}, \nabla u_{t t}^{\eta m}\right)+\left(h^{\prime}\left(u_{t}^{\eta m}\right) u_{t t}^{\eta m}, u_{t t}^{\eta m}\right)_{\Gamma_{1}} \\
=f(t)\left|u_{t t}^{\eta m}\right|^{2}+\left(k(x)\left|u^{\eta m}(t)\right|^{k(x)-1} u_{t}^{\eta m}(t), u_{t t}^{\eta m}\right)_{\Gamma_{1}} \\
+\left(p(x)\left|u^{\eta m}(t)\right|^{p(x)-1} u_{t}^{\eta m}(t), u_{t t}^{\eta m}\right) \\
+\eta f(t)\left(u_{t}^{\eta m}(t), u_{t t}^{\eta m}\right)_{\Gamma_{1}}+g(0) \frac{\mathrm{d}}{\mathrm{d} t}\left(\nabla u^{\eta m}(t), \nabla u_{t}^{\eta m}\right) \\
-g(0)\left|\nabla u_{t}^{\eta m}\right|^{2}+f(t)\left(h\left(u_{t}^{\eta m}\right), u_{t t}^{\eta m}\right)_{\Gamma_{1}} \\
-\left(\int_{0}^{t} g^{\prime \prime}(t-s) \nabla u^{\eta m}(s) \mathrm{d} s, \nabla u_{t}^{\eta m}\right) \\
+\frac{\mathrm{d}}{\mathrm{d} t}\left(\int_{0}^{t} g^{\prime}(t-s) \nabla u^{\eta m}(s) \mathrm{d} s, \nabla u_{t}^{\eta m}\right)-g^{\prime}(0)\left(\nabla u^{\eta m}, \nabla u_{t}^{\eta m}(t)\right) .
\end{gathered}
$$

Next, we are going to analyze the term on the right-hand side of (3.13), taking in mind the estimates (3.9) and (3.10).

Estimate for $I_{1}$ :

$$
\left|I_{1}\right|=\left|f(t)\left(h\left(u_{t}^{\eta m}\right), u_{t t}^{\eta m}\right)_{\Gamma_{1}}\right| \leq \frac{\eta}{8}\left\|u_{t t}^{\eta m}(s)\right\|_{2, \Gamma_{1}}^{2}+\frac{4 L m_{1} m_{2}}{\eta m_{3}} C_{h}\left\|u_{t}^{\eta m}\right\|_{2, \Gamma_{1}}^{2}
$$

Estimate for $I_{2}$ :

$$
\left|I_{2}\right|=\left|-\int_{\Omega} h^{\prime}\left(u_{t}^{\eta m}\right) u_{t}^{\eta m}(t) u_{t t}^{\eta m}(t) \mathrm{d} x\right| \leq \frac{C_{h} C_{1}}{2}+\frac{C_{h}}{2}\left|u_{t t}^{\eta m}(t)\right|^{2}
$$


Estimate for $I_{3}$ : From the generalized Hölder's inequality, Young's inequality and the conditions (2.14), we have

$$
\begin{gathered}
\left|I_{3}\right|=\left|f(t)\left(\left|u^{\eta m}(t)\right|^{k(x)-1} u^{\eta m}(t), u_{t t}^{\eta m}\right)_{\Gamma_{1}}\right| \\
\leq\left(\frac{2 L m_{1} m_{2}}{m_{3}}\right)^{2} C(\varepsilon) \max \left(\int_{\Gamma_{1}}\left|u^{\eta m}\right|^{2 k^{+}} \mathrm{d} \Gamma, \int_{\Gamma_{1}}\left|u^{\eta m}\right|^{2 k^{-}} \mathrm{d} \Gamma\right)+\varepsilon\left\|u_{t t}^{\eta m}\right\|_{2, \Gamma_{1}}^{2} \\
\leq\left(\frac{2 L m_{1} m_{2}}{m_{3}}\right)^{2} C(\varepsilon) \max \left(\left|\nabla u^{\eta m}\right|^{2 k^{+}},\left|\nabla u^{\eta m}\right|^{2 k^{-}}\right)+\varepsilon\left\|u_{t t}^{\eta m}(t)\right\|_{2, \Gamma_{1}}^{2} \\
\leq C_{\varepsilon}+\varepsilon\left\|u_{t t}^{\eta m}(t)\right\|_{2, \Gamma_{1}}^{2} .
\end{gathered}
$$

Estimate for $I_{4}$ : From the generalized Hölder's inequality, it hold that

$$
\begin{gathered}
\left|I_{4}\right|=\left|\left(k(x)\left|u^{\eta m}(t)\right|^{k(x)-1} u_{t}^{\eta m}(t), u_{t t}^{\eta m}\right)_{\Gamma_{1}}\right| \\
\leq k^{+} \max \left(\int_{\Gamma_{1}}\left|u^{\eta m}\right|^{k^{+}-1}\left|u_{t}^{\eta m}\right|\left|u_{t t}^{\eta m}(t)\right| \mathrm{d} \Gamma, \int_{\Gamma_{1}}\left|u^{\eta m}\right|^{k^{-}-1}\left|u_{t}^{\eta m}\right|\left|u_{t t}^{\eta m}(t)\right| \mathrm{d} \Gamma\right) \\
\leq k^{+} \max \left(\begin{array}{c}
\left\|u^{\eta m}(t)\right\|_{2 k^{+}, \Gamma_{1}}^{k^{+}-1}\left\|u_{t}^{\eta m}(t)\right\|_{2 k^{+}, \Gamma_{1}}\left\|u_{t t}^{\eta m}(t)\right\|_{2, \Gamma_{1}}, \\
\left\|u^{\eta m}(t)\right\|_{2 k^{-}, \Gamma_{1}}^{k^{-}}\left\|u_{t}^{\eta m}(t)\right\|_{2 k^{-}, \Gamma_{1}}\left\|u_{t t}^{\eta m}(t)\right\|_{2, \Gamma_{1}}
\end{array}\right) \\
\leq k^{+} \max \left(\left|\nabla u^{\eta m}\right|^{k^{+}-1},\left|\nabla u^{\eta m}\right|^{k^{-}-1}\right)\left|\nabla u_{t}^{\eta m}\right|\left\|u_{t t}^{\eta m}(t)\right\|_{2, \Gamma_{1}} \\
\leq C(\varepsilon)\left|\nabla u_{t}^{\eta m}\right|^{2}+\varepsilon\left\|u_{t t}^{\eta m}(t)\right\|_{2, \Gamma_{1}}^{2}
\end{gathered}
$$

Estimate for $I_{5}$ :

$$
\begin{gathered}
\left|I_{5}\right|=\left|\left(p(x)\left|u^{\eta m}(t)\right|^{p(x)-1} u_{t}^{\eta m}(t), u_{t t}^{\eta m}\right)\right| \\
\leq p^{+} \max \left(\int_{\Omega}\left|u^{\eta m}\right|^{p^{+}-1}\left|u_{t}^{\eta m}\right|\left|u_{t t}^{\eta m}(t)\right| \mathrm{d} x, \int_{\Omega}\left|u^{\eta m}\right|^{p^{-}-1}\left|u_{t}^{\eta m}\right|\left|u_{t t}^{\eta m}(t)\right| \mathrm{d} x\right) \\
\leq p^{+} \max \left(\begin{array}{c}
|| u^{\eta m}(t) \|_{2 p^{+}}^{p^{+}-1}|| u_{t}^{\eta m}(t)||_{2 p^{+}}\left|u_{t t}^{\eta m}(t)\right|, \\
|| u^{\eta m}(t) \|_{2 p^{-}}^{p^{-}}|| u_{t}^{\eta m}(t)||_{2 p^{-}}\left|u_{t t}^{\eta m}(t)\right|
\end{array}\right) \\
\leq p^{+} \max \left(\begin{array}{c}
\left.\left|\nabla u^{\eta m}\right|^{p^{+}-1},\left|\nabla u^{\eta m}\right|^{p^{-}-1}\right)\left|\nabla u_{t}^{\eta m}\right|\left|u_{t t}^{\eta m}(t)\right| \\
\leq C(\varepsilon)\left|\nabla u_{t}^{\eta m}\right|^{2}+\varepsilon\left|u_{t t}^{\eta m}(t)\right|^{2} .
\end{array}\right.
\end{gathered}
$$

Estimate for $I_{6}$ :

$$
\begin{gathered}
\left|I_{6}\right|=\left|f(t)\left(\left|u^{\eta m}(t)\right|^{p(x)-1} u^{\eta m}(t), u_{t t}^{\eta m}\right)\right| \\
\leq \frac{2 L m_{1} m_{2}}{m_{3}} \max \left(\int_{\Omega}\left|u^{\eta m}\right|^{p^{+}}\left|u_{t t}^{\eta m}(t)\right| \mathrm{d} x, \int_{\Omega}\left|u^{\eta m}\right|^{p^{-}}\left|u_{t t}^{\eta m}(t)\right| \mathrm{d} x\right) \\
\leq \max \left(\left|\nabla u^{\eta m}\right|^{p^{+}},\left|\nabla u^{\eta m}\right|^{p^{-}}\right)\left|u_{t t}^{\eta m}(t)\right| \leq C_{\varepsilon}+\varepsilon\left|u_{t t}^{\eta m}(t)\right|^{2}
\end{gathered}
$$


Estimate for $I_{7}$ :

$$
I_{7}=\left|\eta f(t)\left(u_{t}^{\eta m}(t), u_{t t}^{\eta m}\right)_{\Gamma_{1}}\right| \leq \frac{\eta}{8}\left\|u_{t t}^{\eta m}(t)\right\|_{2, \Gamma_{1}}^{2}+2 \eta\left(\frac{2 L m_{1} m_{2}}{m_{3}}\right)^{2}\left\|u_{t}^{\eta m}(t)\right\|_{2, \Gamma_{1}}^{2}
$$

Estimate for $I_{8}$ :

$$
I_{8}=\left|-g^{\prime}(0)\left(\nabla u^{\eta m}, \nabla u_{t}^{\eta m}(t)\right)\right| \leq C_{\varepsilon}+C(\varepsilon)\left|\nabla u_{t}^{\eta m}\right|^{2}
$$

Estimate for $I_{9}$ :

$$
\begin{gathered}
I_{9}=\left|-\left(\int_{0}^{t} g^{\prime \prime}(t-s) \nabla u^{\eta m}(s) \mathrm{d} s, \nabla u_{t}^{\eta m}\right)\right| \leq\left|\nabla u_{t}^{\eta m}\right| \int_{0}^{t} g^{\prime \prime}(t-s)\left|\nabla u^{\eta m}\right| \mathrm{d} s \\
\leq C(\varepsilon)\left|\nabla u_{t}^{m}\right|^{2}+\varepsilon\left\|g^{\prime \prime}\right\|_{L^{1}} \int_{0}^{t}\left|g^{\prime \prime}(t-s)\right|\left|\nabla u^{\eta m}\right|^{2} \mathrm{~d} s \\
\leq C(\varepsilon)\left|\nabla u_{t}^{\eta m}\right|^{2}+\left(\varepsilon\left\|g^{\prime \prime}\right\|_{L^{1}}^{2}+\varepsilon\right) \int_{0}^{t}\left|\nabla u^{\eta m}\right|^{2} \mathrm{~d} s \\
\leq C(\varepsilon)\left|\nabla u_{t}^{\eta m}\right|^{2}+C_{\varepsilon} \sup _{(0, T)}\left\|u^{\eta m}(t)\right\|_{L^{\infty}\left(0, T ; H_{0}^{1}(\Omega)\right)}^{2} .
\end{gathered}
$$

Estimate for $I_{10}$ :

$$
\begin{aligned}
I_{10} & =\left(\int_{0}^{t} g^{\prime}(t-s) \nabla u^{\eta m}(s) \mathrm{d} s, \nabla u_{t}^{\eta m}\right) \\
& \leq \frac{m_{3}}{8}\left|\nabla u^{\eta m}\right|^{2}+\frac{2 \xi(0)\|g\|_{L^{1}}\|g\|_{L^{\infty}}}{m_{3}}\left|\nabla u_{t}^{\eta m}\right|^{2} \\
& \leq \frac{m_{3}}{8}\left|\nabla u_{t}^{\eta m}\right|^{2}+C\left(m_{3}\right) \sup _{(0, T)}\left\|u^{\eta m}(t)\right\|_{L^{\infty}\left(0, T ; H_{0}^{1}(\Omega)\right.}^{2} .
\end{aligned}
$$

By replacing (3.14)-(3.17) in (3.13) and choosing $\varepsilon=\frac{\eta}{4}$, we obtain

$$
\begin{gathered}
\frac{1}{2} \frac{\mathrm{d}}{\mathrm{d} t}\left|u_{t t}^{\eta m}\right|^{2}+\frac{1}{2} M\left(|\nabla \varphi(t)|^{2}\right) \frac{\mathrm{d}}{\mathrm{d} t}\left|\nabla u_{t}^{\eta m}(t)\right|^{2} \\
+g(0)\left|\nabla u_{t}^{\eta m}\right|^{2}+\frac{\eta}{2}\left\|u_{t t}^{\eta m}(t)\right\|_{2, \Gamma_{1}}^{2} \\
\leq-f(t)\left(\int_{0}^{t} g(t-s) \nabla u^{\eta m}(s) \mathrm{d} s, \nabla u_{t t}^{\eta m}\right)+g(0) \frac{\mathrm{d}}{\mathrm{d} t}\left(\nabla u^{\eta m}(t), \nabla u_{t}^{\eta m}\right) \\
+3 C_{\varepsilon}+f(t)\left|u_{t t}^{\eta m}\right|^{2}+3 C(\varepsilon)\left|\nabla u_{t}^{\eta m}\right|^{2}+2 \varepsilon\left|u_{t t}^{\eta m}(t)\right|^{2} \\
+2 \eta\left(\frac{2 L m_{1} m_{2}}{m_{3}}\right)^{2}|| u_{t}^{\eta m}(t) \|_{2, \Gamma_{1}}^{2} \\
+\frac{2 L m_{1} m_{2}}{m_{3}}\left|u_{t t}^{\eta m}(t)\right|^{2}+\frac{4 L m_{1} m_{2}}{\eta m_{3}} C_{h}\left\|u_{t}^{\eta m}\right\|_{2, \Gamma_{1}}^{2} \\
+\frac{C_{h} C_{1}}{2}+\frac{C_{h}}{2}\left|u_{t t}^{\eta m}(t)\right|^{2}+\frac{\mathrm{d}}{\mathrm{d} t}\left(\int_{0}^{t} g^{\prime}(t-s) \nabla u^{\eta m}(s) \mathrm{d} s, \nabla u_{t}^{\eta m}\right) .
\end{gathered}
$$

Employing Hölder's inequality, Young's inequality, integrating by parts on $(0, t)$, the first and second terms on the right-hand side and the first term on the left-hand side 
of (3.19) can be estimated as follows, for

$$
\begin{gathered}
\left|\int_{0}^{t}-f(\zeta)\left(\int_{0}^{\zeta} g(\zeta-s) \nabla u^{\eta m}(s) \mathrm{d} s, \nabla u_{t t}^{\eta m}\right) \mathrm{d} \zeta\right| \\
\leq \frac{2 L m_{1} m_{2}}{m_{3}}\left|\int_{0}^{t}\left(\int_{0}^{\zeta} g(\zeta-s) \nabla u^{\eta m}(s) \mathrm{d} s, \nabla u_{t t}^{\eta m}(\zeta)\right) \mathrm{d} \zeta\right| \\
\leq \frac{2 L m_{1} m_{2}}{m_{3}}\left|\left(\int_{0}^{t} g(t-s) \nabla u^{\eta m}(s) \mathrm{d} s, \nabla u_{t}^{\eta m}(t)\right)\right| \\
+\frac{2 L m_{1} m_{2}}{m_{3}} g(0)\left|\int_{0}^{t}\left(\nabla u^{\eta m}(s), \nabla u_{t}^{\eta m}(s)\right) \mathrm{d} s\right| \\
\leq C+\frac{m_{3}}{8}\left|\nabla u_{t}^{\eta m}\right|^{2}+\frac{L m_{1} m_{2}}{m_{3}} g(0)\left(\int_{0}^{t}\left|\nabla u_{t}^{\eta m}\right|^{2} \mathrm{~d} s+\int_{0}^{t}\left|\nabla u^{\eta m}\right|^{2} \mathrm{~d} s\right) \\
\leq C+\frac{m_{3}}{8}\left|\nabla u_{t}^{\eta m}\right|^{2}+\frac{L m_{1} m_{2}}{m_{3}} g(0) \int_{0}^{t}\left|\nabla u_{t}^{\eta m}\right|^{2} \mathrm{~d} s \\
+\left.\frac{L m_{1} m_{2}}{m_{3}} g(0) \sup _{(0, T)}|| u^{\eta m}(t)\right|_{L^{\infty}} ^{2}\left(0, T ; H_{0}^{1}(\Omega)\right)
\end{gathered}
$$

because, from estimate (3.9) we have

$$
\begin{gathered}
\frac{2 L m_{1} m_{2}}{m_{3}} \int_{\Omega} \nabla u_{t}^{\eta m}(t) \int_{0}^{t} g(t-s) \nabla u^{\eta m}(s) \mathrm{d} s \mathrm{~d} x \leq C\left|\nabla u_{t}^{\eta m}\right|\|g\|_{L^{1}\left(\mathbb{R}_{+}\right)} \\
\leq C+\frac{m_{3}}{8}\left|\nabla u_{t}^{\eta m}\right|^{2},
\end{gathered}
$$

and

$$
\begin{gathered}
g(0) \int_{0}^{t} \frac{\mathrm{d}}{\mathrm{d} t}\left(\nabla u^{\eta m}(t), \nabla u_{t}^{\eta m}\right) \mathrm{d} s \\
\leq \frac{m_{3}}{8}\left|\nabla u_{t}^{\eta m}\right|^{2}+\frac{2}{m_{3}} g(0)^{2}\left|\nabla u^{\eta m}\right|^{2}+g(0)\left|\nabla u_{0}\right|\left|\nabla u_{1}\right| \\
\leq \frac{m_{3}}{8}\left|\nabla u_{t}^{\eta m}\right|^{2}+\left.\frac{2}{m_{3}} g(0)^{2} \sup _{(0, T)}|| u^{\eta m}(t)\right|_{L^{\infty}\left(0, T ; H_{0}^{1}(\Omega)\right)} ^{2}+g(0)\left|\nabla u_{0}\right|\left|\nabla u_{1}\right|
\end{gathered}
$$

and

$$
\begin{gathered}
\frac{1}{2} \int_{0}^{t} M\left(|\nabla \varphi(s)|^{2}\right) \frac{\mathrm{d}}{\mathrm{d} t}\left|\nabla u_{t}^{\eta m}(s)\right|^{2} \mathrm{~d} s \\
\geq\left[\frac{1}{2} M\left(|\nabla \varphi(s)|^{2}\right)\left|\nabla u_{t}^{\eta m}\right|^{2}\right]_{0}^{t}-\frac{1}{2} \int_{0}^{t}\left[\frac{\mathrm{d}^{+}}{\mathrm{d} s} M\left(|\nabla \varphi(s)|^{2}\right)\right]\left|\nabla u_{t}^{\eta m}\right|^{2} \mathrm{~d} s \\
\geq\left[\frac{1}{2} M\left(|\nabla \varphi(s)|^{2}\right)\left|\nabla u_{t}^{\eta m}\right|^{2}\right]_{0}^{t}-L m_{1} m_{2} \int_{0}^{t}\left|\nabla u_{t}^{\eta m}\right|^{2} \mathrm{~d} s, s \in\left[0, T_{1}\right] .
\end{gathered}
$$


Combining, we get

$$
\begin{gathered}
\frac{1}{2}\left|u_{t t}^{\eta m}\right|^{2}+\frac{m_{3}}{8}\left|\nabla u_{t}^{\eta m}\right|^{2}+g(0) \int_{0}^{t}\left|\nabla u_{t}^{\eta m}\right|^{2} \mathrm{~d} s+\eta \int_{0}^{t}|| u_{t t}^{\eta m}(s) \|_{2, \Gamma_{1}}^{2} \\
\leq\left(\frac{2 L m_{1} m_{2}}{m_{3}}+2 \varepsilon+\frac{C_{h}}{2}\right) \int_{0}^{t}\left|u_{t t}^{\eta m}(s)\right|^{2} \mathrm{~d} s \\
+\left(\frac{L m_{1} m_{2}}{m_{3}} g(0)+L m_{1} m_{2}+2 C(\varepsilon)\right) \int_{0}^{t}\left|\nabla u_{t}^{\eta m}\right|^{2} \mathrm{~d} s \\
+\left(\frac{2}{m_{3}} g(0)^{2}+C\left(m_{3}\right)+\frac{L m_{1} m_{2}}{m_{3}} g(0)+C_{\varepsilon}\right) \sup _{(0, T)}\left\|u^{\eta m}(t)\right\|_{L^{\infty}}^{2}\left(0, T ; H_{\Gamma_{0}}^{1}(\Omega)\right) \\
+\left(\frac{4 L m_{1} m_{2}}{\eta m_{3}} C_{h}+2 \eta\left(\frac{2 L m_{1} m_{2}}{m_{3}}\right)^{2}\right) \int_{0}^{t}\left\|u_{t}^{\eta m}(t)\right\|_{2, \Gamma_{1}}^{2} \mathrm{~d} s+C_{5}
\end{gathered}
$$

where

$$
C_{5}=\left(C, C_{h}, C_{1}, u_{1}, u_{0}, C_{\varepsilon}, T, g(0), \frac{L m_{1} m_{2}}{m_{3}}\right) .
$$

Choosing $\varepsilon=\frac{\eta}{4}$, therefore, by using estimates (3.10), (3.5) and Gronwall's lemma, we arrive at

$$
\left|u_{t t}^{\eta m}\right|^{2}+\left|\nabla u_{t}^{\eta m}\right|^{2}+\int_{0}^{t}\left|\nabla u_{t}^{\eta m}\right|^{2} \mathrm{~d} s+\int_{0}^{t}\left\|u_{t t}^{\eta m}(s)\right\|_{2, \Gamma_{1}}^{2} \mathrm{~d} s \leq C_{6}
$$

where $C_{6}$ is a positive constant which is independent of $m, \eta$ and $t$.

Thanks to (3.10) and (3.20), we obtain

$$
\left(u^{\eta m}\right) \text { is a bounded sequence in } L^{\infty}\left(0, T_{0} ; H_{\Gamma_{0}}^{1}(\Omega)\right) \text {, }
$$

$\left(u_{t}^{\eta m}\right)$ is a bounded sequence in $L^{\infty}\left(0, T_{0} ; H_{\Gamma_{0}}^{1}(\Omega)\right) \cap L^{2}\left(0, T_{0} ; L^{2}(\Omega)\right)$,

$$
\left(u_{t t}^{\eta m}\right) \text { is bounded in } L^{\infty}\left(0, T_{0} ; L^{2}(\Omega),\right.
$$

$\left(u_{t}^{\eta m}\right)$ is a bounded sequence in $L^{2}\left(0, T_{0} ; L^{2}\left(\Gamma_{1}\right)\right)$,

$$
\left(u_{t t}^{\eta m}\right) \text { is bounded in } L^{2}\left(0, T_{0} ; L^{2}\left(\Gamma_{1}\right)\right) \text {, }
$$

By (2.11), (3.22) and (3.24), we have

$$
h\left(u_{t}^{\eta m}\right) \text { is bounded in } L^{2}\left(0, T_{0} ; L^{2}\left(\Gamma_{1}\right)\right) \text {. }
$$

From (3.21)-(3.24), there exists a subsequence of $\left(u^{\eta m}\right)$, still denote by $\left(u^{\eta m}\right)$, such that such that

$$
\begin{aligned}
& u^{\eta m} \longrightarrow u^{\eta} \text { weak star in } L^{\infty}\left(0, T_{0} ; H_{\Gamma_{0}}^{1}(\Omega)\right), \\
& u_{t}^{\eta m} \longrightarrow u_{t}^{\eta} \text { weak star in } L^{\infty}\left(0, T_{0} ; H_{\Gamma_{0}}^{1}(\Omega)\right), \\
& u_{t t}^{\eta m} \longrightarrow u_{t t}^{\eta} \text { weak star in } L^{\infty}\left(0, T_{0} ; L^{2}(\Omega),\right. \\
& u_{t}^{\eta m} \longrightarrow u_{t}^{\eta} \text { weakly in } L^{2}\left(0, T_{0} ; L^{2}\left(\Gamma_{1}\right)\right), \\
& u_{t t}^{\eta m} \longrightarrow u_{t t}^{\eta} \text { weakly in } L^{2}\left(0, T_{0} ; L^{2}\left(\Gamma_{1}\right)\right), \\
& u_{t}^{\eta m} \longrightarrow u_{t}^{\eta} \text { weak star in } L^{\infty}\left(0, T_{0} ; H^{\frac{1}{2}}\left(\Gamma_{1}\right)\right),
\end{aligned}
$$


Since $H^{\frac{1}{2}}\left(\Gamma_{1}\right) \hookrightarrow L^{2}\left(\Gamma_{1}\right)$ and $H^{1}\left(\Gamma_{0}\right) \hookrightarrow L^{2}(\Omega)$ are compact and from Aubin-Lions theorem, we deduce that

$$
\begin{aligned}
& u^{\eta m} \longrightarrow u^{\eta} \text { strongly in } L^{2}\left(0, T_{0} ; L^{2}(\Omega)\right), \\
& u^{\eta m} \longrightarrow u^{\eta} \text { strongly in } L^{2}\left(0, T_{0} ; L^{2}\left(\Gamma_{1}\right)\right), \\
& u_{t}^{\eta m} \longrightarrow u_{t}^{\eta} \text { strongly in } L^{2}\left(0, T_{0} ; L^{2}(\Omega)\right) \\
& u_{t}^{\eta m} \longrightarrow u_{t}^{\eta} \text { strongly in } L^{2}\left(0, T_{0} ; L^{2}\left(\Gamma_{1}\right)\right),
\end{aligned}
$$

Consequently, by making use of Lions' Lemma [27, Lemma 1.3.], we have

$$
\begin{aligned}
& \left|u^{\eta m}(t)\right|^{p(.)-1} u^{\eta m}(t) \rightarrow\left|u^{\eta}(t)\right|^{p(.)-1} u^{\eta}(t) \text { weakly in } L^{2}\left(0, T_{0} ; L^{2}(\Omega)\right) \\
& \left|u^{\eta m}(t)\right|^{k(.)-1} u^{\eta m}(t) \rightarrow\left|u^{\eta}(t)\right|^{k(.)-1} u^{\eta}(t) \text { weakly in } L^{2}\left(0, T_{0} ; L^{2}\left(\Gamma_{1}\right)\right) .
\end{aligned}
$$

From (3.28) and (3.29) and since the injection of $H^{\frac{1}{2}}\left(\Gamma_{1}\right)$ in $L^{2}\left(\Gamma_{1}\right)$ is compact, there exists a subsequence of $\left(u^{\eta m}\right)$, still denote by $\left(u^{\eta m}\right)$, such that

$$
u_{t}^{\eta m} \longrightarrow u_{t}^{\eta} \text { a.e. in } Q_{0},
$$

where $\left.Q_{0}=\Gamma_{1} \times\right] 0, T_{0}[$. Then by $(2.11)$, we have

$$
h\left(u_{t}^{\eta m}\right) \rightarrow h\left(u_{t}^{\eta}\right) \text { a.e. in } Q_{0},
$$

From (3.25) and (3.32) and by using Lions' lemma, we conclude that

$$
h\left(u_{t}^{\eta m}\right) \rightarrow h\left(u_{t}^{\eta}\right) \text { weakly in } L^{2}\left(0, T_{0} ; L^{2}\left(\Gamma_{1}\right)\right)
$$

The convergences $(3.26),(3.28),(3.31),(4.16)$ and $(3.33)$ permit us to pass to the limit in the (3.3). Since $\left(w_{j}\right)$ is a basis of $H_{\Gamma_{0}}^{1}(\Omega) \cap H^{2}(\Omega)$ and $V_{m}$ is dense in $H_{\Gamma_{0}}^{1}(\Omega) \cap H^{2}(\Omega)$, after passing to the limit, we obtain

$$
\begin{gathered}
\int_{0}^{T_{0}}\left(u_{t t}^{\eta}(t), v\right) \theta(t) \mathrm{d} t+\int_{0}^{T_{0}} M\left(|\nabla \varphi(t)|^{2}\right)\left(\nabla u^{\eta}, \nabla v\right) \theta(t) \mathrm{d} t \\
-\int_{0}^{T_{0}}\left(\int_{0}^{t} g(t-s) \nabla u^{\eta}(s) \mathrm{d} s, \nabla v\right) \theta(t) \mathrm{d} t+\int_{0}^{T_{0}}\left(h\left(u_{t}^{\eta}\right), v\right)_{\Gamma_{1}} \theta(t) \mathrm{d} t \\
+\eta \int_{0}^{T_{0}}\left(u_{t}^{\eta}(t), v\right)_{\Gamma_{1}} \theta(t) \mathrm{d} t=\int_{0}^{T_{0}}\left(\left|u^{\eta}(t)\right|^{k(x)-1} u^{\eta}(t), v\right)_{\Gamma_{1}} \theta(t) \mathrm{d} t \\
+\int_{0}^{T_{0}}\left(\left|u^{\eta}(t)\right|^{p(x)-1} u^{\eta}(t), v\right) \theta(t) \mathrm{d} t,
\end{gathered}
$$

for all $\theta \in D(0, T)$, and for all $v \in H_{\Gamma_{0}}^{1}(\Omega) \cap H^{2}(\Omega)$.

We can see that the estimates (3.10) and (3.21) are also independent of $\eta$. Therefore, by the same argument used to obtain $u^{\eta}$ from $u^{\eta m}$, we can pass to the limit when 
$\eta \rightarrow 0$ in $u^{\eta}$, obtaining a function $u$ such that

$$
\begin{gathered}
u^{\eta} \longrightarrow u \text { weak star in } L^{\infty}\left(0, T_{0} ; H_{\Gamma_{0}}^{1}(\Omega)\right), \\
u_{t}^{\eta} \longrightarrow u_{t} \text { weak star in } L^{\infty}\left(0, T_{0} ; H_{\Gamma_{0}}^{1}(\Omega)\right), \\
u_{t t}^{\eta} \longrightarrow u_{t t} \text { weak star in } L^{\infty}\left(0, T_{0} ; L^{2}(\Omega),\right. \\
u_{t}^{\eta} \longrightarrow u_{t} \text { weak star in } L^{\infty}\left(0, T_{0} ; H^{\frac{1}{2}}\left(\Gamma_{1}\right)\right), \\
h\left(u_{t}^{\eta}\right) \rightarrow h\left(u_{t}\right) \text { weakly in } L^{2}\left(0, T_{0} ; L^{2}\left(\Gamma_{1}\right)\right), \\
\left|u^{\eta}(t)\right|^{p(.)-1} u^{\eta}(t) \rightarrow|u(t)|^{p(.)-1} u(t) \text { weakly in } L^{2}\left(0, T_{0} ; L^{2}(\Omega)\right), \\
\left|u^{\eta}(t)\right|^{k(.)-1} u^{\eta}(t) \rightarrow|u(t)|^{k(.)-1} u(t) \text { weakly in } L^{2}\left(0, T_{0} ; L^{2}\left(\Gamma_{1}\right)\right)
\end{gathered}
$$

From the above convergence in $(3.10)$ and by observing that $V_{m}$ is dense in $H_{\Gamma_{0}}^{1}(\Omega) \cap$ $H^{2}(\Omega)$, we have

$$
\begin{gathered}
\int_{0}^{T_{0}}\left(u_{t t}(t), v\right) \theta(t) \mathrm{d} t+\int_{0}^{T_{0}} M\left(|\nabla \varphi(t)|^{2}\right)(\nabla u, \nabla v) \theta(t) \mathrm{d} t \\
-\int_{0}^{T_{0}}\left(\int_{0}^{t} g(t-s) \nabla u(s) \mathrm{d} s, \nabla v\right) \theta(t) \mathrm{d} t+\int_{0}^{T_{0}}\left(h\left(u_{t}\right), v\right)_{\Gamma_{1}} \theta(t) \mathrm{d} t \\
=\int_{0}^{T_{0}}\left(|u(t)|^{k(x)-1} u(t), v\right)_{\Gamma_{1}} \theta(t) \mathrm{d} t+\int_{0}^{T_{0}}\left(|u(t)|^{p(x)-1} u(t), v\right) \theta(t) \mathrm{d} t,
\end{gathered}
$$

for all $v \in H_{\Gamma_{0}}^{1}(\Omega)$ and for all $\theta \in D\left(0, T_{0}\right)$.

By taking $v \in D(\Omega)$, we get that

$$
\frac{\partial^{2} u}{\partial t^{2}}-M\left(|\nabla \varphi(t)|^{2}\right) \Delta u+\int_{0}^{t} g(t-s) \Delta u(s) \mathrm{d} s=|u|^{p(x)-1} u \text { in } D^{\prime}(\Omega) .
$$

Therefore, by (3.36) and (3.37), we obtain

$$
\frac{\partial^{2} u}{\partial t^{2}}-M\left(|\nabla \varphi(t)|^{2}\right) \Delta u+\int_{0}^{t} g(t-s) \Delta u(s) \mathrm{d} s=|u|^{p(x)-1} u \text { in } L^{2}\left(0, T_{0} ; L^{2}(\Omega)\right) \text {. }
$$

From the hypotheses of $M, g$ and (3.35), we conclude that

$$
g(t-s) u, M\left(|\nabla \varphi(t)|^{2}\right) u \in L^{\infty}\left(0, T_{0} ; H_{\Gamma_{0}}^{1}(\Omega)\right),
$$

and by (3.39),

$$
-\Delta\left(M\left(|\nabla \varphi(t)|^{2}\right) u-\int_{0}^{t} g(t-s) u(s) \mathrm{d} s\right) \in L^{2}\left(0, T_{0} ; L^{2}(\Omega)\right)
$$

Then

$$
M\left(|\nabla \varphi(t)|^{2}\right) \frac{\partial u}{\partial \nu}-\int_{0}^{t} g(t-s) \frac{\partial}{\partial \nu} u(s) \mathrm{d} s \in L^{2}\left(0, T_{0} ; H^{-\frac{1}{2}}\left(\Gamma_{1}\right)\right)
$$


according to Miranda [29] is established. By taking (3.39) into account and making use of the generalized Green formula, we deduce

$$
M\left(|\nabla \varphi(t)|^{2}\right) \frac{\partial u}{\partial \nu}-\int_{0}^{t} g(t-s) \frac{\partial}{\partial \nu} u(s) \mathrm{d} s+h\left(u_{t}\right)=|u|^{k(x)-1} u
$$

in $D^{\prime}\left(0, T_{0} ; H^{-\frac{1}{2}}\left(\Gamma_{1}\right)\right)$, and as $h\left(u_{t}\right),|u|^{k(.)-1} u \in L^{2}\left(0, T_{0} ; L^{2}\left(\Gamma_{1}\right)\right)$, we infer

$M\left(|\nabla \varphi(t)|^{2}\right) \frac{\partial u}{\partial \nu}-\int_{0}^{t} g(t-s) \frac{\partial}{\partial \nu} u(s) \mathrm{d} s+h\left(u_{t}\right)=|u|^{k(x)-1} u$ in $L^{2}\left(0, T_{0} ; L^{2}\left(\Gamma_{1}\right)\right)$.

Prove the uniqueness of the local solution. To this end let $u(t)$ and $v(t)$ be two local solutions to (3.3) with the same initial value. Let $w(t)=u(t)-v(t)$. Then $w(0)=0$, $w_{t}(0)=0$ for all $t \in\left[0, T_{0}\right]$ and

$$
\begin{gathered}
\left(w^{\prime \prime}(t), \psi\right)+M\left(|\nabla \varphi(t)|^{2}\right)(\nabla w, \nabla \psi)-\left(\int_{0}^{t} g(t-s) \nabla w(s) \mathrm{d} s, \nabla \psi\right) \\
+\left(h\left(u_{t}\right)-h\left(v_{t}\right), \psi\right)_{\Gamma_{1}}=\left(|u(t)|^{k(x)-1} u(t)-|v(t)|^{k(x)-1} v(t), \psi\right)_{\Gamma_{1}} \\
+\left(|u(t)|^{p(x)-1} u(t)-|v(t)|^{p(x)-1} v(t), \psi\right)
\end{gathered}
$$

for all $\psi \in H_{\Gamma_{0}}^{1}(\Omega)$. By replacing $\psi=w_{t}(t)$ in (3.41) and observing that $\left(h\left(u_{t}\right)-h\left(v_{t}\right), \psi\right)_{\Gamma_{1}} \geq 0$, it hold that

$$
\begin{gathered}
\frac{1}{2} \frac{\mathrm{d}}{\mathrm{d} t}\left|w_{t}(t)\right|^{2}+\frac{1}{2} \frac{\mathrm{d}^{+}}{\mathrm{d} t}\left(\left(M\left(|\nabla \varphi(t)|^{2}\right)-\int_{0}^{t} g(s) \mathrm{d} s\right)|\nabla w(t)|^{2}\right) \\
+\frac{1}{2} \frac{\mathrm{d}}{\mathrm{d} t}(g \diamond \nabla w)(t)-\frac{1}{2}\left(g^{\prime} \diamond \nabla w\right)(t)+\frac{1}{2} g(t)|\nabla w(t)|^{2} \\
\leq \frac{1}{2}\left(\frac{\mathrm{d}^{+}}{\mathrm{d} t} M\left(|\nabla \varphi(t)|^{2}\right)\right)|\nabla w|^{2}+\left(|u(t)|^{k(x)-1} u(t)-|v(t)|^{k(x)-1} v(t), w_{t}(t)\right)_{\Gamma_{1}} \\
+\left(|u(t)|^{p(x)-1} u(t)-|v(t)|^{p(x)-1} v(t), w_{t}(t)\right)
\end{gathered}
$$

From the generalized Hölder's and Young's inequalities and estimates (3.21)-(3.24), it hold that

$$
\begin{aligned}
& \left|\left(|u(t)|^{k(x)-1} u(t)-|v(t)|^{k(x)-1} v(t), w_{t}\right)\right| \\
& \leq c \max \left(\begin{array}{c}
\left(\|u(t)\|_{2 k^{-}}^{k^{-}-1}+\|v(t)\|_{2 k^{-}}^{k^{-}-1}\right)\|u(t)-v(t)\|_{2 k^{-}}\left\|w_{t}\right\|_{2}, \\
\left(\|u(t)\|_{2 k^{+}}^{k^{+}-1}+\|v(t)\|_{2 k^{+}}^{k^{+}-1}\right)\|u(t)-v(t)\|_{2 k^{+}}\left\|w_{t}\right\|_{2}
\end{array}\right) \\
& \leq c c_{*} \max \left(\begin{array}{c}
\left(|\nabla u(t)|^{k^{-}-1}+|\nabla v(t)|^{k^{-}-1}\right), \\
\left(|\nabla u(t)|^{k^{+}-1}+|\nabla v(t)|^{k^{+}-1}\right)
\end{array}\right)|\nabla w|\left|w_{t}\right| \\
& \leq c|\nabla w|^{2}+c\left|w_{t}\right|^{2} \text {. }
\end{aligned}
$$


By the same manner

$$
\begin{aligned}
& \left|\left(|u(t)|^{p(x)-1} u(t)-|v(t)|^{p(x)-1} v(t), w_{t}\right)\right|
\end{aligned}
$$

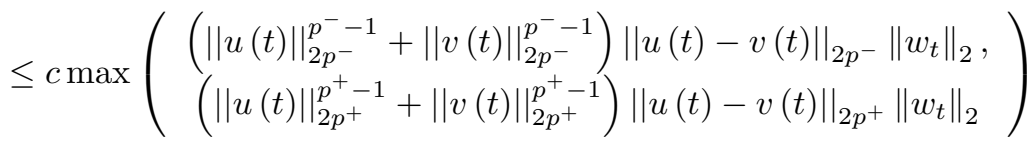

$$
\begin{aligned}
& \leq c c_{*} \max \left(\begin{array}{c}
\left(|\nabla u(t)|^{p^{-}-1}+|\nabla v(t)|^{p^{-}-1}\right) \\
\left(|\nabla u(t)|^{p^{+}-1}+|\nabla v(t)|^{p^{+}-1}\right)
\end{array}\right)|\nabla w|\left|w_{t}\right| \\
& \leq c|\nabla w|^{2}+c\left|w_{t}\right|^{2} .
\end{aligned}
$$

Substituting the last two inequalities in (3.42) and integrating the results over $(0, t)$, it holds

$$
\frac{1}{2}\left|w_{t}(t)\right|^{2}+\frac{1}{2} l|\nabla w(t)|^{2} \leq C \int_{0}^{t}\left(|\nabla w|^{2}+\left|w_{t}\right|^{2}\right) \mathrm{d} s
$$

Thus, employing Gronwall's lemma, we conclude that $\left|w_{t}(t)\right|^{2}=|\nabla w(t)|^{2}=0$.

Consequently this completes the proof of the lemma.

We are concerned with the existence and uniqueness of local solution in time to degenerate wave equation (1.1)-(1.4). So by using Lemma 3.1 we prove the existence and uniqueness of local solution in time to (1.1)-(1.4) by the Banach fixed point theorem.

Theorem 3.2. Assume that $M(r)>0$ is a locally Lipschitz function and assume that the following condition is satisfied

$$
\begin{gathered}
1<k^{+}<\frac{n-1}{n-2} \text { and } 1<p^{+} \leq \frac{n}{n-2} \text { if } n \geq 3, \\
1 \leq k^{-} \leq k^{+}<\infty \text { and } 1 \leq p^{-} \leq p^{+}<\infty \text { if } n=2 .
\end{gathered}
$$

Let $\left(u_{0}, u_{1}\right) \in H_{\Gamma_{0}}^{1}(\Omega) \cap H^{2}(\Omega) \times H_{\Gamma_{0}}^{1}(\Omega)$ with $\left|\nabla u_{1}\right| \neq 0$ or $\left|\nabla u_{0}\right| \neq 0$. Assume that $M\left(\left|\nabla u_{0}\right|^{2}\right)>0$. Then there exists a time $T_{0}>0$ and a unique local weak solution $u(t)$ to (1.1)-(1.4) with the initial value $\left(u_{0}, u_{1}\right)$ satisfying

$$
\begin{gathered}
u(t) \in C\left(\left[0, T_{0}\right]: H_{\Gamma_{0}}^{1}(\Omega)\right), \\
u_{t}(t) \in C\left(\left[0, T_{0}\right]: L^{2}(\Omega)\right) \cap C\left(\left[0, T_{0}\right]: H_{\Gamma_{0}}^{1}(\Omega)\right), \\
u_{t t}(t) \in C\left(\left[0, T_{0}\right]: L^{2}(\Omega)\right) .
\end{gathered}
$$

Proof. Since $M\left(\left|\nabla u_{0}\right|^{2}\right)>0$, there exists a positive real number $m_{3}$ such that $0<$ $m_{3}<M\left(\left|\nabla u_{0}\right|^{2}\right)$. Assume that

$$
0<m_{3}-\int_{0}^{+\infty} g(t) \mathrm{d} t<1
$$


Let $R_{0}$ be a positive real number such that

$$
R_{0}=\sqrt{\frac{2}{l}\left(\left|\nabla u_{1}\right|^{2}+M\left(\left|\nabla u_{0}\right|^{2}\right)\left|\nabla u_{0}\right|^{2}\right)}
$$

Since $M\left(\left|\nabla u_{0}\right|^{2}\right)>0$, for sufficiently small time $T>0$, we define the space $B_{T}\left(R_{0}\right)$ by

$$
B_{T}\left(R_{0}\right)=\left\{\begin{array}{c}
\phi(t) \in C\left([0, T]: H_{\Gamma_{0}}^{1}(\Omega)\right) \cap C\left([0, T]: H_{\Gamma_{0}}^{1}(\Omega)\right), \\
\phi^{\prime}(t) \in C\left([0, T]: L^{2}(\Omega) \cap C\left([0, T]: H_{\Gamma_{0}}^{1}(\Omega)\right),\right. \\
\phi^{\prime \prime}(t) \in C\left([0, T]: L^{2}(\Omega)\right), \\
M\left(|\nabla \phi(t)|^{2}\right) \geq m_{3},\left|\nabla \phi^{\prime}(t)\right|^{2}+|\nabla \phi(t)|^{2} \leq R_{0}^{2} \text { on }[0, T], \\
\phi(0)=u_{0}, \phi^{\prime}(0)=u_{1} .
\end{array}\right\}
$$

We introduce the metric $\mathrm{d}$ on the space $B_{T}\left(R_{0}\right)$ by

$$
\mathrm{d}(u, v)=\sup _{0 \leq t \leq T}\left(\left|u_{t}(t)-v_{t}(t)\right|^{2}+|\nabla u(t)-\nabla v(t)|^{2}\right) \text { for } u, v \in B_{T}\left(R_{0}\right) .
$$

Then the space $B_{T}\left(R_{0}\right)$ is the complete metric space. Let $\phi \in B_{T}\left(R_{0}\right)$.

Then $|\nabla \phi(t)| \leq R_{0},\left|\nabla \phi^{\prime}(t)\right| \leq R_{0}$ and $M\left(|\nabla \phi(t)|^{2}\right) \geq m_{3}$ for all $t \in[0, T]$. Thus thanks to Lemma 3.1 we obtain a unique local weak solution $u(t)$ on $\left[0, T_{1}\right]$ with $T_{1} \leq T$ to the following wave equation:

$$
\begin{aligned}
\left(u_{t t}(t), v\right)+M\left(|\nabla \varphi(t)|^{2}\right)(\nabla u, \nabla v)-\left(\int_{0}^{t} g(t-s) \nabla u(s) \mathrm{d} s, \nabla v\right)+\left(h\left(u_{t}\right), v\right)_{\Gamma_{1}} \\
=\left(|u(t)|^{k(x)-1} u(t), v\right)_{\Gamma_{1}}+\left(|u(t)|^{p(x)-1} u(t), v\right) \\
\quad \operatorname{in} L^{2}\left(0, T_{1} ; H^{-1}(\Omega)\right) \cap L^{2}\left(0, T_{1} ; H^{-\frac{1}{2}}\left(\Gamma_{1}\right)\right) .
\end{aligned}
$$

Let $T=T_{1}$ without loss of generality. Define the mapping $\Phi$ by

$$
\Phi(\varphi)=u
$$

Then we have that

$$
\begin{gathered}
\Phi(\varphi)=u \in B_{T}\left(R_{0}\right) \text { for } \varphi \in B_{T}\left(R_{0}\right), \\
\Phi: B_{T}\left(R_{0}\right) \rightarrow B_{T}\left(R_{0}\right) \text { is a contractive mapping. }
\end{gathered}
$$

For showing (3.44), posing $v=u_{t}$ in (3.43) and taking

$$
\left(h\left(u_{t}\right), u_{t}\right)_{\Gamma_{1}}-\frac{1}{2}\left(g^{\prime} \diamond \nabla u\right)(t)+\frac{1}{2} g(t)|\nabla u(t)|^{2} \geq 0,
$$


into account we have that:

$$
\begin{gathered}
\frac{1}{2} \frac{\mathrm{d}^{+}}{\mathrm{d} t}\left(\left|u_{t}(t)\right|^{2}+\left(\left(M\left(|\nabla \varphi(t)|^{2}\right)-\int_{0}^{t} g(s) \mathrm{d} s\right)|\nabla u(t)|^{2}\right)+\frac{1}{2}(g \diamond \nabla u)(t)\right) \\
\leq \frac{1}{2}\left(\frac{\mathrm{d}^{+}}{\mathrm{d} t} M\left(|\nabla \varphi(t)|^{2}\right)\right)|\nabla u|^{2} \\
+\left(|u(t)|^{k(x)-1} u(t), u_{t}\right)_{\Gamma_{1}}+\left(|u(t)|^{p(x)-1} u(t), u_{t}\right)=I_{1}+I_{2}+I_{3} .
\end{gathered}
$$

And so we estimates $I_{1}$ and $I_{2}$ as follows

$$
I_{1}=\frac{1}{2}\left(\frac{\mathrm{d}^{+}}{\mathrm{d} t} M\left(|\nabla \varphi(t)|^{2}\right)\right)|\nabla u|^{2} \leq L|\nabla \varphi(t)|\left|\nabla \varphi^{\prime}(t)\right||\nabla u|^{2} \leq \frac{L R_{0}^{2}}{l} \psi_{\varphi} u(t)
$$

Taking estimates (4.9) into account

$$
\begin{gathered}
\left|I_{2}\right|=\left|\left(k(x)|u(t)|^{k(x)-1} u(t), u_{t}\right)_{\Gamma_{1}}\right| \\
\leq k^{+} \max \left(\int_{\Gamma_{1}}|u|^{k^{+}}\left|u_{t}(t)\right| \mathrm{d} \Gamma, \int_{\Gamma_{1}}|u|^{k^{-}}\left|u_{t}\right| \mathrm{d} \Gamma\right) \\
\leq k^{+} \max \left(\|u(t)\|_{2 k^{+}, \Gamma_{1}}^{k^{+}},\|u(t)\|_{2 k^{-}, \Gamma_{1}}^{k^{-}}\right)\left\|u_{t}(t)\right\|_{2, \Gamma_{1}} \\
\leq k^{+} \max \left(B_{*}^{k^{+}}|\nabla u|^{k^{+}}, B_{*}^{k^{-}}|\nabla u|^{k^{-}}\right)\left\|u_{t}(t)\right\|_{2, \Gamma_{1}} \\
\leq k^{+} \max \left(\left(B_{*} R_{0}\right)^{k^{+}},\left(B_{*} R_{0}\right)^{k^{-}}\right)\left\|u_{t}(t)\right\|_{2, \Gamma_{1}} \leq C_{2}
\end{gathered}
$$

similarly

$$
\begin{gathered}
\left|I_{3}\right|=\left|\left(p(x)|u(t)|^{p(x)-1} u(t), u_{t t}^{\eta m}\right)\right| \\
\leq p^{+} \max \left(\int_{\Omega}|u|^{p^{+}}\left|u_{t}(t)\right| \mathrm{d} x, \int_{\Omega}|u|^{p^{-}}\left|u_{t}(t)\right| \mathrm{d} x\right) \\
\leq p^{+} \max \left(\left.|| u(t)\right|_{2 p^{+}} ^{p^{+}},\left.|| u(t)\right|_{2 p^{-}} ^{p}\right)\left|u_{t}(t)\right| \\
\leq p^{+} \max \left(B^{p^{+}}|\nabla u|^{p^{+}}, B^{p^{-}}|\nabla u|^{p^{-}}\right)\left|u_{t}(t)\right| \\
\leq p^{+} \max \left(\left(B R_{0}\right)^{p^{+}},\left(B R_{0}\right)^{p^{-}}\right)\left|u_{t}(t)\right| \leq C_{3} \psi_{\varphi} u(t)^{\frac{1}{2}}
\end{gathered}
$$

because $\left\|u_{t}(t)\right\|_{2, \Gamma_{1}} \leq C\left|\nabla u_{t}(t)\right|$ is bounded on $[0, T]$ by Lemma 3.1. Thus

$$
\frac{\mathrm{d}^{+}}{\mathrm{d} t} \psi_{\varphi} u(t) \leq 2 C_{2}+2 C_{1} \psi_{\varphi} u(t)+2 C_{3} \psi_{\varphi} u(t)^{\frac{1}{2}}
$$

where

$$
\psi_{\varphi} u(t)=\left|u_{t}(t)\right|^{2}+\left(\left(M\left(|\nabla \varphi(t)|^{2}\right)-\int_{0}^{t} g(s) \mathrm{d} s\right)|\nabla u(t)|^{2}\right)+(g \diamond \nabla u)(t),
$$


and $C_{1}=\frac{L R_{0}^{2}}{l}$. Gronwall inequality yields

$$
\begin{aligned}
\psi_{\varphi} u(t) & \leq\left(\psi_{\varphi} u(0)+2 C_{2} T_{2}\right) e^{\left(2 C_{1}+2 C_{3}\right) T_{2}} \\
& <l R_{0}^{2}, \quad 0 \leq t \leq T_{2},
\end{aligned}
$$

for sufficiently small $0<T_{2} \leq T_{1}$. Thus

$$
\begin{gathered}
l R_{0}^{2}>\left|u_{t}(t)\right|^{2}+\left(\left(M\left(|\nabla \varphi(t)|^{2}\right)-\int_{0}^{t} g(s) \mathrm{d} s\right)|\nabla u(t)|^{2}\right)+(g \diamond \nabla u)(t) \\
>\left|u_{t}(t)\right|^{2}+l|\nabla u(t)|^{2}, \quad(l<1)
\end{gathered}
$$

We have that

$$
R_{0}^{2}>\left|u_{t}(t)\right|^{2}+|\nabla u(t)|^{2}, 0 \leq t \leq T_{2},
$$

Let $T=T_{2}$ be modified. Thus (3.44) is satisfied. Rest to show (3.45). Let $w=u_{1}-u_{2}$, where $u_{1}=\Phi\left(\varphi_{1}\right), u_{2}=\Phi\left(\varphi_{2}\right)$ with $\varphi_{1}, \varphi_{2} \in B_{T}\left(R_{0}\right)$. Then we have that

$$
\begin{gathered}
\left(w_{t t}(t), v\right)+M\left(\left|\nabla \varphi_{1}(t)\right|^{2}\right)(\nabla w, \nabla v)+\left(h\left(u_{1 t}\right)-h\left(u_{2 t}\right), v\right)_{\Gamma_{1}} \\
=\left(M\left(\left|\nabla \varphi_{2}(t)\right|^{2}\right)-M\left(\left|\nabla \varphi_{1}(t)\right|^{2}\right)\right)\left(\nabla u_{2}, \nabla v\right) \\
\quad+\left(\int_{0}^{t} g(t-s) \nabla w(s) \mathrm{d} s, \nabla v\right) \\
=\left(\left|u_{1}(t)\right|^{k(x)-1} u_{1}(t)-\left|u_{2}(t)\right|^{k(x)-1} u_{2}(t), v\right)_{\Gamma_{1}} \\
+\left(\left|u_{1}(t)\right|^{p(x)-1} u_{1}(t)-\left|u_{2}(t)\right|^{p(x)-1} u_{2}(t), v\right) \text { in } L^{2}\left(0, T_{1} ; H^{-1}(\Omega)\right) .
\end{gathered}
$$

Set

$$
\beta_{\varphi_{1}}(w)(t)=\left|w_{t}(t)\right|^{2}+\left(\left(M\left(\left|\nabla \varphi_{1}(t)\right|^{2}\right)-\int_{0}^{t} g(s) \mathrm{d} s\right)|\nabla w(t)|^{2}\right)
$$

Since $0<l=m_{3}-\int_{0}^{\infty} g(s) \mathrm{d} s<1$, we have that

$$
\beta_{\varphi_{1}}(w)(t) \geq l\left(\left|w_{t}(t)\right|^{2}+|\nabla w(t)|^{2}\right)
$$

By replacing $v$ in (3.46) by $w_{t}$ we have that

$$
\begin{gathered}
\frac{1}{2} \frac{\mathrm{d}^{+}}{\mathrm{d} t}\left(\left|w_{t}(t)\right|^{2}+\left(\left(M\left(\left|\nabla \varphi_{1}(t)\right|^{2}\right)-\int_{0}^{t} g(s) \mathrm{d} s\right)|\nabla w(t)|^{2}\right)\right) \\
+\frac{1}{2} \frac{\mathrm{d}}{\mathrm{d} t}(g \diamond \nabla w)(t)-\frac{1}{2}\left(g^{\prime} \diamond \nabla w\right)(t)+\frac{1}{2} g(t)|\nabla u(t)|^{2} \\
\quad \leq \frac{1}{2}\left(\frac{\mathrm{d}^{+}}{\mathrm{d} t} M\left(\left|\nabla \varphi_{1}(t)\right|^{2}\right)\right)|\nabla w|^{2} \\
+\left(M\left(\left|\nabla \varphi_{2}(t)\right|^{2}\right)-M\left(\left|\nabla \varphi_{1}(t)\right|^{2}\right)\right)\left(\nabla u_{2}, \nabla w_{t}\right) \\
+\left(\left|u_{1}(t)\right|^{k(x)-1} u_{1}(t)-\left|u_{2}(t)\right|^{k(x)-1} u_{2}(t), w_{t}\right)_{\Gamma_{1}} \\
+\left(\left|u_{1}(t)\right|^{p(x)-1} u_{1}(t)-\left|u_{2}(t)\right|^{p(x)-1} u_{2}(t), w_{t}\right)=I_{4}+I_{5}+I_{6}+I_{7}
\end{gathered}
$$


Then

$$
\begin{gathered}
\left|I_{4}\right|=\left.\left.\left|\frac{1}{2}\left(\frac{\mathrm{d}^{+}}{\mathrm{d} t} M\left(\left|\nabla \varphi_{1}(t)\right|^{2}\right)\right)\right| \nabla w\right|^{2}\left|\leq L R_{0}^{2}\right| \nabla w\right|^{2} \\
\leq \frac{L R_{0}^{2}}{l} \beta_{\varphi_{1}}(w)(t):=\xi_{4} \beta_{\varphi_{1}}(w)(t)
\end{gathered}
$$

and

$$
\begin{gathered}
\left|I_{5}\right|=\left|\left(M\left(\left|\nabla \varphi_{2}(t)\right|^{2}\right)-M\left(\left|\nabla \varphi_{1}(t)\right|^{2}\right)\right)\left(\nabla u_{2}, \nabla w_{t}\right)\right| \\
\leq L R_{0}^{2} \mathrm{~d}\left(\varphi_{1}, \varphi_{2}\right)^{\frac{1}{2}}\left|\nabla u_{2}\right|\left|\nabla w_{t}\right| \leq \frac{2 L R_{0}^{2}}{\sqrt{l}} \mathrm{~d}\left(\varphi_{1}, \varphi_{2}\right)^{\frac{1}{2}} \beta_{\varphi_{1}}(w)(t)^{\frac{1}{2}} \\
:=\xi_{5} \mathrm{~d}\left(\varphi_{1}, \varphi_{2}\right)^{\frac{1}{2}} \beta_{\varphi_{1}}(w)(t)^{\frac{1}{2}} .
\end{gathered}
$$

Since

$$
\begin{gathered}
\left|I_{6}\right|=\left|\left(\left|u_{1}(t)\right|^{k(x)-1} u_{1}(t)-\left|u_{2}(t)\right|^{k(x)-1} u_{2}(t), w_{t}\right)_{\Gamma_{1}}\right| \\
\leq c \max \left(\begin{array}{c}
\left(|| u_{1}(t) \|_{2 k^{-}}^{k^{-}-1}+\left.|| u_{2}(t)\right|_{2 k^{-}} ^{k^{-}-1}\right)\left\|u_{1}(t)-u_{2}(t)||_{2 k^{-}}\right\| w_{t} \|_{2}, \\
\left(\left.\left\|\left.u_{1}(t)\right|_{2 k^{+}} ^{k^{+}-1}+\right\| u_{2}(t)\right|_{2 k^{+}} ^{k^{+}-1}\right)|| u_{1}(t)-u_{2}(t)\left\|_{2 k^{+}}\right\| w_{t} \|_{2}
\end{array}\right) \\
\leq c c_{*} \max \left(\begin{array}{c}
\left(\left|\nabla u_{1}(t)\right|^{k^{-}-1}+\left|\nabla u_{2}(t)\right|^{k^{-}-1}\right), \\
\left(\left|\nabla u_{1}(t)\right|^{k^{+}-1}+\left|\nabla u_{2}(t)\right|^{k^{+}-1}\right)
\end{array}\right)|\nabla w|\left|w_{t}\right| \\
\leq 2 c c_{*}\left(\sqrt{C_{1}^{k^{-}-1}}+\sqrt{C_{1}^{k^{+}-1}}\right)|\nabla w|\left|w_{t}\right| \\
\leq c c_{*} \frac{1}{l}\left(\sqrt{C_{1}^{k^{-}-1}}+\sqrt{C_{1}^{k^{+}-1}}\right) \beta_{\varphi_{1}}(w)(t):=\zeta_{6} \beta_{\varphi_{1}}(w)(t)
\end{gathered}
$$

and

$$
\begin{gathered}
\left|I_{7}\right|=\left|\left(\left|u_{1}(t)\right|^{p(x)-1} u_{1}(t)-\left|u_{2}(t)\right|^{p(x)-1} u_{2}(t), w_{t}\right)\right| \\
\leq c \max \left(\begin{array}{c}
\left(\left\|u_{1}(t)\right\|_{2 p^{-}}^{p^{-}-1}+\left\|u_{2}(t)\right\|_{2 p^{-}}^{p^{-}-1}\right)\left\|u_{1}(t)-u_{2}(t)\right\|_{2 p^{-}}\left\|w_{t}\right\|_{2}, \\
\left(\left.\left\|\left.u_{1}(t)\right|_{2 p^{+}} ^{p^{+}-1}+\right\| u_{2}(t)\right|_{2 p^{+}} ^{k^{+}-1}\right)|| u_{1}(t)-u_{2}(t)\left\|_{2 p^{+}}\right\| w_{t} \|_{2}
\end{array}\right) \\
\leq c c_{*} \max \left(\begin{array}{c}
\left(\left|\nabla u_{1}(t)\right|^{p^{-}-1}+\left|\nabla u_{2}(t)\right|^{p^{-}-1}\right), \\
\left(\left|\nabla u_{1}(t)\right|^{p^{+}-1}+\left|\nabla u_{2}(t)\right|^{p^{+}-1}\right)
\end{array}\right)|\nabla w|\left|w_{t}\right| \\
\leq 2 c c_{*}\left(\sqrt{C_{1}^{p^{-}-1}}+\sqrt{C_{1}^{p^{+}-1}}\right)|\nabla w|\left|w_{t}\right| \\
\leq c c_{*} \frac{1}{l}\left(\sqrt{C_{1}^{p^{-}-1}}+\sqrt{C_{1}^{p^{+}-1}}\right) \beta_{\varphi_{1}}(w)(t):=\zeta_{7} \beta_{\varphi_{1}}(w)(t)
\end{gathered}
$$

It follows that

$$
\beta_{\varphi_{1}}(w)(t) \leq\left(\xi_{4}+\zeta_{6}+\zeta_{7}\right) \int_{0}^{t} \beta_{\varphi_{1}}(w)(s) \mathrm{d} s+\xi_{5} \int_{0}^{t} \mathrm{~d}\left(\varphi_{1}, \varphi_{2}\right)^{\frac{1}{2}} \beta_{\varphi_{1}}(w)(s)^{\frac{1}{2}} \mathrm{~d} s
$$


Gronwall's lemma gives

$$
\mathrm{d}\left(u_{1}, u_{2}\right) \leq \frac{\xi_{5}^{2} T}{l} \mathrm{~d}\left(\varphi_{1}, \varphi_{2}\right) e^{\left(1+\xi_{4}+\zeta_{6}+\zeta_{7}\right) T} .
$$

Choose a $0<T_{3} \leq T$ small enough which satisfies that

$$
\frac{\xi_{5}^{2}}{l} T_{3} e^{\left(1+\xi_{4}+\zeta_{6}+\zeta_{7}\right) T_{3}}<1
$$

Thus by the Banach contraction mapping theorem there exists a fixed point

$$
u=\Phi(u) \in B_{T_{3}}\left(R_{0}\right),
$$

which is a unique local weak solution in time to (1.1)-(1.4). This completes the proof of the theorem.

\section{Uniform decay rates}

In this section, we shall prove the general decay rates of solution for system (1.1)-(1.4).

In this section we assume that

$$
\begin{aligned}
& M\left(|\nabla u|^{2}\right)=m_{3}+b|\nabla u|^{2}+\sigma \int_{\Omega} \nabla u \nabla u_{t} \mathrm{~d} x, \\
& m_{3}>0, b>0, \sigma: \text { positive and small enough. }
\end{aligned}
$$

and providing that $h$ satisfies:

$\left(\mathbf{H}^{\prime} \mathbf{3}\right)$ Hypotheses on $h . h: \mathbb{R} \rightarrow \mathbb{R}$ is a non-decreasing function with $h(s) s \geq 0$ for all $s \in \mathbb{R}$ and there exists a convex and increasing function $H: \mathbb{R}_{+} \rightarrow \mathbb{R}_{+}$ of class $C^{1}\left(\mathbb{R}_{+}\right) \cap C^{2}((0, \infty))$ satisfying $H(0)=0$ and $H$ is linear on $[0, r]$ or $H^{\prime}(0)=0$ and $H^{\prime \prime}>0$ on $(0, r](r>0)$ such that

$$
\begin{gathered}
m_{1}|s| \leq|h(s)| \leq M_{1}|s| \text { if }|s| \geq r \\
h^{2}(s) \leq H^{-1}(s h(s)) \text { if }|s| \leq r
\end{gathered}
$$

where $r, m_{1}$ and $M_{1}$ are positive constants.

For formulate our results it is convenient to introduce the energy of the system

$$
E(t)=\frac{1}{2}\left|u_{t}(t)\right|^{2}+J(u(t)) \text { for } u \in H_{\Gamma_{0}}^{1}(\Omega)
$$

where

$$
\begin{aligned}
J(u(t))= & \frac{1}{2}\left(m_{3}-\int_{0}^{t} g(s) \mathrm{d} s\right)\|\nabla u(t)\|_{2}^{2}+\frac{b}{4}\|\nabla u\|_{2}^{4}+\frac{1}{2}(g \circ \nabla(u))(t) \\
& -\int_{\Omega} \frac{1}{p(x)+1}|u|^{p(x)+1} \mathrm{~d} x-\int_{\Gamma_{1}} \frac{1}{k(x)+1}|u|^{k(x)+1} \mathrm{~d} \Gamma,
\end{aligned}
$$


so, we have

$$
\begin{aligned}
J(u(t)) \geq \frac{1}{2} & \left(m_{3}-\int_{0}^{t} g(s) \mathrm{d} s\right)\|\nabla u(t)\|_{2}^{2}+\frac{b}{4}\|\nabla u\|_{2}^{4}+\frac{1}{2}(g \circ \nabla(u))(t) \\
& -\frac{1}{p^{-}+1} \max \left(\int_{\Omega}|u|^{p^{+}+1} \mathrm{~d} x, \int_{\Omega}|u|^{p^{-}+1} \mathrm{~d} x\right) \\
& -\frac{1}{k^{-}+1} \max \left(\int_{\Gamma_{1}}|u|^{k^{+}+1} \mathrm{~d} \Gamma, \int_{\Gamma_{1}}|u|^{k^{-}+1} \mathrm{~d} \Gamma\right) \\
& \geq \frac{1}{2} l\|\nabla u(t)\|_{2}^{2}+\frac{b}{4}\|\nabla u\|_{2}^{4}+\frac{1}{2}(g \circ \nabla(u))(t) \\
- & \left(\frac{1}{p^{-}+1} \int_{\Omega}|u|^{p^{+}+1} \mathrm{~d} x+\frac{1}{k^{-}+1} \int_{\Gamma_{1}}|u|^{k^{+}+1} \mathrm{~d} \Gamma\right) \\
- & \left(\frac{1}{p^{-}+1} \int_{\Omega}|u|^{p^{-}+1} \mathrm{~d} x+\frac{1}{k^{-}+1} \int_{\Gamma_{1}}|u|^{k^{-}+1} \mathrm{~d} \Gamma\right)
\end{aligned}
$$

then

$$
E^{\prime}(t)=-\sigma\left(\frac{1}{2} \frac{\mathrm{d}}{\mathrm{d} t}\|\nabla u(t)\|_{2}^{2}\right)^{2}-\int_{\Gamma_{1}} u_{t} h\left(u_{t}\right) \mathrm{d} \Gamma+\frac{1}{2}\left(g^{\prime} \circ \nabla(u)\right)(t)-\frac{1}{2} g(t)\|\nabla u(t)\|_{2}^{2} \leq 0,
$$

so the energy $E(t)$ is nonincreasing function.

Next, with some modifications, we define a functionals $F_{1,2}$ introduced by Cavalcanti et al. [28], which helps in establishing desired results. Setting

$$
\begin{aligned}
& F_{1}(x)=\frac{1}{4} x^{2}-\frac{K_{-, \Omega}^{p^{-}+1}}{p^{-}+1} x^{p^{-}+1}-\frac{K_{-, \Gamma}^{k^{-}+1}}{k^{-}+1} x^{k^{-}+1}, x>0 \\
& F_{2}(x)=\frac{1}{4} x^{2}-\frac{K_{+, \Omega}^{p^{+}+1}}{p^{-}+1} x^{p^{+}+1}-\frac{K_{+, \Gamma}^{k^{+}+1}}{k^{-}+1} x^{k^{+}+1}, x>0,
\end{aligned}
$$

where

$$
\begin{aligned}
& 0<K_{+, \Omega}=\sup _{u \in H_{\Gamma_{0}}^{1}(\Omega), u \neq 0}\left(\frac{\|u\|_{p^{+}+1}}{\sqrt{l\|\nabla u\|_{2}^{2}+\frac{b}{2}\|\nabla u\|_{2}^{4}}}\right)<\infty, \\
& 0<K_{-, \Omega}=\sup _{u \in H_{\Gamma_{0}}^{1}(\Omega), u \neq 0}\left(\frac{\|u\|_{p^{-}+1}}{\sqrt{l\|\nabla u\|_{2}^{2}+\frac{b}{2}\|\nabla u\|_{2}^{4}}}\right)<\infty .
\end{aligned}
$$

and

$$
\begin{gathered}
0<K_{+, \Gamma}=\sup _{u \in H_{\Gamma_{0}}^{1}(\Omega), u \neq 0}\left(\frac{\|u\|_{k^{+}+1, \Gamma_{1}}}{\sqrt{l\|\nabla u\|_{2}^{2}+\frac{b}{2}\|\nabla u\|_{2}^{4}}}\right)<\infty, \\
K_{-, \Gamma}=\sup _{u \in H_{\Gamma_{0}}^{1}(\Omega), u \neq 0}\left(\frac{\|u\|_{k^{-}+1, \Gamma_{1}}}{\sqrt{l\|\nabla u\|_{2}^{2}+\frac{b}{2}\|\nabla u\|_{2}^{4}}}\right)<\infty .
\end{gathered}
$$


Remark 4.1. (i). As in [28], we can verify that the functional $F_{1}$ is increasing in $\left(0, \lambda_{1}\right)$, decreasing in $\left(\lambda_{1}, \infty\right)$, and $F_{1}$ has a maximum at $\lambda_{1}$ with the maximum value

$$
\mathrm{d}_{1}=F_{1}\left(\lambda_{1}\right)=\frac{1}{4} \lambda_{1}^{2}-\frac{K_{-, \Omega}^{p^{-}+1}}{p^{-}+1} \lambda_{1}^{p^{-}+1}-\frac{K_{-, \Gamma}^{k^{-}+1}}{k^{-}+1} \lambda_{1}^{k^{-}+1},
$$

also, for $F_{2}$ is increasing in $\left(0, \lambda_{2}\right)$, decreasing in $\left(\lambda_{2}, \infty\right)$, and $F_{2}$ has a maximum at $\lambda_{2}$ with the maximum value

$$
\mathrm{d}_{2}=F_{2}\left(\lambda_{2}\right)=\frac{1}{4} \lambda_{2}^{2}-\frac{K_{+, \Omega}^{p^{+}+1}}{p^{-}+1} \lambda_{2}^{p^{+}+1}-\frac{K_{+, \Gamma}^{k^{+}+1}}{k^{-}+1} \lambda_{2}^{k^{+}+1},
$$

$\lambda_{1}$ and $\lambda_{2}$ are the first positive zero of the derivative functions $F_{1}^{\prime}(x)$ and $F_{2}^{\prime}(x)$, respectively.

(ii). From $(4.3),(4.5),(2.9),(2.12)$ and the definition of $F_{1}$ and $F_{2}$ we have

$$
\begin{gathered}
E(t) \geq J(t) \geq \frac{1}{4} \gamma(t)^{2}-\frac{K_{-, \Omega}^{p^{-}+1}}{p^{-}+1} \gamma(t)^{p^{-}+1}-\frac{K_{-, \Gamma}^{k^{-}+1}}{k^{-}+1} \gamma(t)^{k^{-}+1} \\
+\frac{1}{4} \gamma(t)^{2}-\frac{K_{+, \Omega}^{p^{+}+1}}{p^{-}+1} \gamma(t)^{p^{+}+1}-\frac{K_{+, \Gamma}^{k^{+}+1}}{k^{-}+1} \gamma(t)^{k^{+}+1}=F_{1}(\gamma(t))+F_{2}(\gamma(t)), t \geq 0,
\end{gathered}
$$

where

$$
\gamma(t)=\sqrt{l\|\nabla u\|_{2}^{2}+\frac{b}{2}\|\nabla u\|_{2}^{4}+(g \circ \nabla(u))(t)}
$$

Now, if one considers $\gamma(t)<\lambda_{0}=\min \left(\lambda_{1}, \lambda_{2}\right)$, then, from (4.15), we get

$$
\begin{gathered}
E(t) \geq F_{1}(\gamma(t))+F_{2}(\gamma(t)) \\
>\gamma(t)^{2}\left(\frac{1}{4}-\frac{K_{-, \Omega}^{p^{-}+1}}{p^{-}+1} \gamma(t)^{p^{-}-1}-\frac{K_{-, \Gamma}^{k^{-}+1}}{k^{-}+1} \gamma(t)^{k^{-}-1}\right) \\
+\gamma(t)^{2}\left(\frac{1}{4}-\frac{K_{+, \Omega}^{p^{+}+1}}{p^{-}+1} \gamma(t)^{p^{+}-1}-\frac{K_{+, \Gamma}^{k^{+}+1}}{k^{-}+1} \gamma(t)^{k^{+}-1}\right), t \geq 0,
\end{gathered}
$$

which together with the identities

$$
\begin{gathered}
\frac{1}{2}-K_{-, \Omega}^{p^{-}+1} \gamma(t)^{p^{-}-1}-K_{-, \Gamma}^{k^{-}+1} \gamma(t)^{k^{-}-1}=0, \text { and } \\
\frac{1}{2}-\frac{p^{+}+1}{p^{-}+1} K_{+, \Omega}^{p^{+}+1} \gamma(t)^{p^{+}-1}-\frac{k^{+}+1}{k^{-}+1} K_{+, \Gamma}^{k^{+}+1} \gamma(t)^{k^{+}-1}=0
\end{gathered}
$$

give

$$
F_{1}(\gamma(t))>c_{0} \gamma(t)^{2}, c_{0}=\left\{\begin{array}{l}
\frac{p^{-}-1}{4\left(p^{-}+1\right)} \text { if } k^{-} \geq p^{-} \\
\frac{k^{-}-1}{4\left(k^{-}+1\right)} \text { if } p^{-} \geq k^{-}
\end{array}\right.
$$


also, since $\frac{p^{+}+1}{p^{-}+1}>1$ and $\frac{k^{+}+1}{k^{-}+1}>1$ and from (4.17) we deduce that

$$
\begin{gathered}
0=\frac{1}{2}-\frac{p^{+}+1}{p^{-}+1} K_{+, \Omega}^{p^{+}+1} \gamma(t)^{p^{+}-1}-\frac{k^{+}+1}{k^{-}+1} K_{+, \Gamma}^{k^{+}+1} \gamma(t)^{k^{+}-1} \\
\leq \frac{1}{2}-K_{+, \Omega}^{p^{+}+1} \gamma(t)^{p^{+}-1}-K_{+, \Gamma}^{k^{+}+1} \gamma(t)^{k^{+}-1}
\end{gathered}
$$

therefore

$$
-K_{+, \Omega}^{p^{+}+1} \gamma(t)^{p^{+}-1}-K_{+, \Gamma}^{k^{+}+1} \gamma(t)^{k^{+}-1} \geq-\frac{1}{2}
$$

and consequently,

$$
\begin{gathered}
F_{2}(t)>\gamma(t)^{2}\left(\frac{1}{4}-\frac{K_{+, \Omega}^{p^{+}+1}}{p^{-}+1} \gamma(t)^{p^{+}-1}-\frac{K_{+, \Gamma}^{k^{+}+1}}{k^{-}+1} \gamma(t)^{k^{+}-1}\right) \\
>c_{0} \gamma(t)^{2}, \quad c_{0}=\left\{\begin{array}{l}
\frac{p^{-}-1}{4\left(p^{-}+1\right)} \text { if } k^{-} \geq p^{-} \\
\frac{k^{-}-1}{4\left(k^{-}+1\right)} \text { if } p^{-} \geq k^{-}
\end{array}\right.
\end{gathered}
$$

consequently

$$
E(t) \geq F_{1}(\gamma(t))+F_{2}(\gamma(t))=F(\gamma(t)) \geq 2 c_{0} \gamma(t)^{2}
$$

and identities (4.16), (4.17) are derived because $\lambda_{1}$ and $\lambda_{2}$ are the first positive zero of the derivative function $F_{1}^{\prime}(x)$ and $F_{2}^{\prime}(x)$ respectively.

Lemma 4.2. Let $\left(u_{0}, u_{1}\right) \in H_{\Gamma_{0}}^{1}(\Omega) \times H_{\Gamma_{0}}^{1}(\Omega)$ and hypotheses $\left(H_{1}\right)-\left(H_{3}\right)$ hold. Assume further that $\gamma(0)=\sqrt{l\left\|\nabla u_{0}\right\|_{2}^{2}+\frac{b}{2}\left\|\nabla u_{0}\right\|_{2}^{4}}<\lambda_{0}$ and $E(0)<\mathrm{d}=\min \left(\mathrm{d}_{1}, \mathrm{~d}_{2}\right)$. Then

$$
\gamma(t)=\sqrt{l\|\nabla u\|_{2}^{2}+\frac{b}{2}\|\nabla u\|_{2}^{4}+(g \circ \nabla(u))(t)}<\lambda_{0},
$$

for all $t \in[0, T)$.

Proof. Using (4.15) and considering $E(t)$ is a non-increasing function, we obtain

$$
F(\gamma(t))=F_{1}(\gamma(t))+F_{2}(\gamma(t)) \leq E(t) \leq E(0)<\mathrm{d}, t \in[0, T)
$$

In addition, from Remark $4.1(i)$, we see that $F$ is increasing in $\left(0, \lambda_{0}\right)$, decreasing in $\left(\max \left(\lambda_{1}, \lambda_{2}\right), \infty\right)$, and $F \rightarrow-\infty$ as $\max \left(\lambda_{1}, \lambda_{2}\right) \rightarrow \infty$. Thus, as $E(0)<\mathrm{d}$, there exist $0 \leq \lambda_{3}^{\prime} \leq \lambda_{0} \leq \lambda_{3}$ such that $F\left(\lambda_{3}^{\prime}\right)=F\left(\lambda_{3}\right)=E(0)$. Besides, through the assumption $\gamma(0)<\lambda_{0}$, we observe for $t=0$ that

$$
F(\gamma(0)) \leq E(0)=F\left(\lambda_{3}^{\prime}\right) .
$$

This implies that $\gamma(0) \leq \lambda_{3}^{\prime}$. Next, we will prove that

$$
\gamma(t) \leq \lambda_{3}^{\prime}, t \in[0, T) \text {. }
$$

To establish (4.21), we reason by absurd. Suppose that (4.21) does not hold, then there exists $t^{*} \in(0, T)$ such that $\gamma\left(t^{*}\right)>\lambda_{3}^{\prime}$.

Case 1. If $\lambda_{3}^{\prime}<\gamma\left(t^{*}\right)<\lambda_{0}$, then

$$
F\left(\gamma\left(t^{*}\right)\right)>F\left(\lambda_{3}^{\prime}\right)=E(0) \geq E\left(t^{*}\right) .
$$

This contradicts (4.20). 
Case 2. If $\gamma\left(t^{*}\right) \geq \lambda_{0}$, then by continuity of $\gamma(t)$, there exists $0<t_{1}<t^{*}$ such that

$$
\lambda_{3}^{\prime}<\gamma\left(t_{1}\right)<\lambda_{0}
$$

then

$$
F\left(\gamma\left(t_{1}\right)\right)>F\left(\lambda_{3}^{\prime}\right)=E(0) \geq E\left(t_{1}\right) .
$$

This is also a contradiction of (4.20). Thus, we have proved (4.21).

Theorem 4.3. Under the hypotheses of Lemma 4.2 the problem (1.1)-(1.4) have a global solution.

Proof. It follows from (4.19), (4.18) and (4.15) that

$$
\frac{1}{2}\left|u_{t}\right|^{2}+2 c_{0} \gamma(t)^{2} \leq \frac{1}{2}\left|u_{t}\right|^{2}+F(\gamma(t)) \leq \frac{1}{2}\left|u_{t}\right|^{2}+J(t)=E(t)<E(0)<\mathrm{d} .
$$

Thus, we establish the boundedness of $u_{t}$ in $L^{2}(\Omega)$ and the boundedness of $u$ in $H_{\Gamma_{0}}^{1}$. Moreover, from (2.13), (2.14) and (4.22), we also obtain

$$
\begin{gathered}
\int_{\Omega} \frac{1}{p(x)+1}|u|^{p(x)+1} \mathrm{~d} x+\int_{\Gamma_{1}} \frac{1}{k(x)+1}|u|^{k(x)+1} \mathrm{~d} \Gamma \\
\leq \frac{1}{p^{-}+1} \max \left(\int_{\Omega}|u|^{p^{+}+1} \mathrm{~d} x, \int_{\Omega}|u|^{p^{-}+1} \mathrm{~d} x\right) \\
\quad+\frac{1}{k^{-}+1} \max \left(\int_{\Gamma_{1}}|u|^{k^{+}+1} \mathrm{~d} \Gamma, \int_{\Gamma_{1}}|u|^{k^{-}+1} \mathrm{~d} \Gamma\right) \\
\leq \frac{1}{p^{-}+1} \max \left(B^{p^{+}+1}|\nabla u|^{p^{+}-1}, B^{p^{-}+1}|\nabla u|^{p^{-}-1}\right)|\nabla u|^{2} \\
+\frac{1}{k^{-}+1} \max \left(B_{*}^{k^{+}+1}|\nabla|^{k^{+}-1}, B_{*}^{k^{-}+1}|\nabla u|^{k^{-}-1}\right)|\nabla u|^{2} \\
\leq L l|\nabla|^{2} \leq \frac{L}{2 c_{0}} E(t)<\frac{L}{2 c_{0}} E(0)<\frac{L}{2 c_{0}} \mathrm{~d}
\end{gathered}
$$

which implies that the boundedness of $u$ in $L^{p(.)+1}(\Omega)$ and in $L^{k(.)+1}\left(\Gamma_{1}\right)$ with

$$
\begin{aligned}
L & =\frac{1}{l}\left(\frac{1}{p^{-}+1} \max \left(B^{p^{+}+1}\left(\frac{E(0)}{2 l c_{0}}\right)^{p^{+}-1}, B^{p^{-}+1}\left(\frac{E(0)}{2 l c_{0}}\right)^{p^{-}-1}\right)\right) \\
& +\frac{1}{l}\left(\frac{1}{k^{-}+1} \max \left(B_{*}^{k^{+}+1}\left(\frac{E(0)}{2 l c_{0}}\right)^{k^{+}-1}, B_{*}^{k^{-}+1}\left(\frac{E(0)}{2 l c_{0}}\right)^{k^{-}-1}\right)\right) .
\end{aligned}
$$

Hence, it must have $T=\infty$.

Now, we shall investigate the asymptotic behavior of the energy function $E(t)$. First, let us define the perturbed modified energy by

$$
G(t)=M E(t)+\varepsilon \Phi(t)+\Psi(t)
$$

where

$$
\Phi(t)=\int_{\Omega} u_{t} u \mathrm{~d} x+\frac{\sigma}{4}\|\nabla u(t)\|_{2}^{4},
$$




$$
\Psi(t)=\int_{\Omega} u_{t} \int_{0}^{t} g(t-s)(u(s)-u(t)) \mathrm{d} s \mathrm{~d} x
$$

and $M, \varepsilon$ are some positive constants to be specified later.

In order to prove the main theorem, we recall the following lemmas.

Lemma 4.4. There exist two positive constants $\beta_{1}$ and $\beta_{2}$ such that

$$
\beta_{1} E(t) \leq G(t) \leq \beta_{2} E(t)
$$

relation holds, for $\varepsilon>0$ small enough while $M>0$ is large enough.

Proof. By Hölder's and Young's inequalities, (2.9) and (2.12), we deduce that

$$
\begin{gathered}
|G(t)-M E(t)| \leq \varepsilon|\Phi(t)|+|\Psi(t)| \\
\leq \frac{\varepsilon+1}{2}\left|u_{t}\right|^{2}+\frac{\varepsilon B^{2}}{2}|\nabla u|^{2}+\frac{\sigma \varepsilon}{4}|\nabla u|^{4}+\frac{1}{2} \int_{\Omega}\left(\int_{0}^{t} g(t-s)(u(s)-u(t)) \mathrm{d} s\right)^{2} \mathrm{~d} x \\
\leq \frac{\varepsilon+1}{2}\left|u_{t}\right|^{2}+\frac{\varepsilon B^{2}}{2}|\nabla u|^{2}+\frac{\sigma \varepsilon}{4}|\nabla u|^{4}+\frac{B^{2}\left(m_{3}-l\right)}{2}(g \diamond \nabla u)(t) \\
\leq c_{1}\left(\frac{1}{2}\left|u_{t}\right|^{2}+2 c_{0}\left(l|\nabla u|^{2}+(g \diamond \nabla u)(t)+\frac{b}{2}|\nabla u|^{4}\right)\right)
\end{gathered}
$$

where

$$
c_{1}=\max \left(\varepsilon+1, \frac{\varepsilon B^{2}}{8 c_{0} l}, \frac{B^{2}\left(m_{3}-l\right)}{8 c_{0} l}, \frac{\sigma \varepsilon}{8 b c_{0}}\right) .
$$

Employing (4.22) and choosing $\varepsilon>0$ small enough and $M$ sufficiently large, there exist two positive constants $\beta_{1}$ and $\beta_{2}$ such that

$$
\beta_{1} E(t) \leq G(t) \leq \beta_{2} E(t) .
$$

Lemma 4.5. Assume that the hypotheses of Lemma 4.2 be fulfilled. Furthermore, if $E(0)$ is small enough, then, for any $t_{0}>0$, the functional $G(t)$ verifies, along solution of (1.1)-(1.4) and for $t \geq t_{0}$,

$$
G^{\prime}(t) \leq-\alpha_{1} E(t)+\alpha_{2}(g \diamond \nabla u)(t)+\alpha_{3} \int_{\Gamma_{1}} h^{2}\left(u_{t}\right) \mathrm{d} \Gamma-\alpha_{4} E(0) E^{\prime}(t)
$$

where $\alpha_{i}, i=1, \ldots, 4$ are some positive constants.

Proof. In the following, we estimate the derivative of $G(t)$. From (4.24) and (1.1)(1.4), we have

$$
\begin{gathered}
\Phi^{\prime}(t)=\left|u_{t}\right|^{2}-\left(m_{3}+b|\nabla u|^{2}\right)+\int_{\Omega} \nabla u(t) \int_{0}^{t} g(t-s) \nabla u(s) \mathrm{d} s \mathrm{~d} x-\int_{\Gamma_{1}} u h\left(u_{t}\right) \mathrm{d} \Gamma \\
+\int_{\Omega}|u|^{p(x)+1} \mathrm{~d} x+\int_{\Gamma_{1}}|u|^{k(x)+1} \mathrm{~d} \Gamma .
\end{gathered}
$$

Employing Hölder's inequality, Young's inequality, (2.14) and (2.9), the third and fourth terms on the right-hand side of (4.28) can be estimated as follows, for $\eta, \delta>0$,

$$
\left|\int_{\Omega} \nabla u(t) \int_{0}^{t} g(t-s) \nabla u(s) \mathrm{d} s \mathrm{~d} x\right| \leq\left(\eta+m_{3}-l\right)|\nabla u|^{2}+\frac{\left(m_{3}-l\right)}{4 \eta}(g \diamond \nabla u)(t),
$$


and

$$
\left|\int_{\Gamma_{1}} u h\left(u_{t}\right) \mathrm{d} \Gamma\right| \leq \delta B_{*}^{2}|\nabla u|^{2}+\frac{1}{4 \delta} \int_{\Gamma_{1}} h^{2}\left(u_{t}\right) \mathrm{d} \Gamma .
$$

A substitution of (4.29)-(4.30) into (4.28) yields

$$
\begin{gathered}
\Phi^{\prime}(t)=\left|u_{t}\right|^{2}-\left(-\eta+l-\delta B_{*}^{2}\right)|\nabla u|^{2}+\frac{\left(m_{3}-l\right)}{4 \eta}(g \diamond \nabla u)(t)-\int_{\Gamma_{1}} u h\left(u_{t}\right) \mathrm{d} \Gamma \\
+\frac{1}{4 \delta} \int_{\Gamma_{1}} h^{2}\left(u_{t}\right) \mathrm{d} \Gamma+\int_{\Omega}|u|^{p(x)+1} \mathrm{~d} x+\int_{\Gamma_{1}}|u|^{k(x)+1} \mathrm{~d} \Gamma
\end{gathered}
$$

Letting $\eta=\frac{l}{2}>0$ and $\delta=\frac{l}{4 B_{*}^{2}}$ in above inequality, we obtain

$$
\begin{aligned}
\Phi^{\prime}(t) \leq & \left|u_{t}\right|^{2}-\frac{l}{4}|\nabla u|^{2}+\frac{\left(m_{3}-l\right)}{2 l}(g \diamond \nabla u)(t)-\int_{\Gamma_{1}} u h\left(u_{t}\right) \mathrm{d} \Gamma \\
& +\frac{B_{*}^{2}}{l} \int_{\Gamma_{1}} h^{2}\left(u_{t}\right) \mathrm{d} \Gamma+\int_{\Omega}|u|^{p(x)+1} \mathrm{~d} x+\int_{\Gamma_{1}}|u|^{k(x)+1} \mathrm{~d} \Gamma .
\end{aligned}
$$

For estimate $\Psi^{\prime}(t)$, taking the derivative of $\Psi(t)$ in (4.25) and using (1.1)-(1.4), we obtain

$$
\begin{gathered}
\Psi^{\prime}(t)=\int_{\Omega}\left(m_{3}+b|\nabla u|^{2}\right) \nabla u(t) \int_{0}^{t} g(t-s)(\nabla u(t)-\nabla u(s)) \mathrm{d} s \mathrm{~d} x \\
+\int_{\Omega}\left(\sigma \int_{\Omega} \nabla u \nabla u_{t} \mathrm{~d} x\right) \nabla u(t) \int_{0}^{t} g(t-s)(\nabla u(t)-\nabla u(s)) \mathrm{d} s \mathrm{~d} x \\
-\int_{\Omega}\left(\int_{0}^{t} g(t-s) \nabla u(s) \mathrm{d} s\right)\left(\int_{0}^{t} g(t-s)(\nabla u(t)-\nabla u(s)) \mathrm{d} s\right) \mathrm{d} x \\
\quad+\int_{\Gamma_{1}} h\left(u_{t}\right) \int_{0}^{t} g(t-s)(u(t)-u(s)) \mathrm{d} s \mathrm{~d} \Gamma \\
-\int_{\Gamma_{1}}|u|^{k(x)-1} u \int_{0}^{t} g(t-s)(u(t)-u(s)) \mathrm{d} s \mathrm{~d} \Gamma \\
-\int_{\Omega}|u|^{p(x)-1} u \int_{0}^{t} g(t-s)(u(t)-u(s)) \mathrm{d} s \mathrm{~d} x \\
-\int_{\Omega} u_{t} \int_{0}^{t} g^{\prime}(t-s)(u(t)-u(s)) \mathrm{d} s \mathrm{~d} x-\left(\int_{0}^{t} g(s) \mathrm{d} s\right)\left|u_{t}\right|^{2} .
\end{gathered}
$$

Similar to deriving (4.31), in what follows we will estimate the right-hand side of (4.32). Using Young's inequality, Hölder's inequality,

$$
\begin{gathered}
|\nabla u|^{2} \leq \frac{E(0)}{2 l c_{0}} \text { by }(4.22), \\
E^{\prime}(t) \leq-\sigma\left(\frac{1}{2} \frac{\mathrm{d}}{\mathrm{d} t}\|\nabla u(t)\|_{2}^{2}\right)^{2} \text { by }(4.6),
\end{gathered}
$$


and applying (2.14) and (2.9), we have, for $\delta>0$,

$$
\begin{aligned}
& \left|\int_{\Omega}\left(m_{3}+b|\nabla u|^{2}\right) \nabla u(t) \int_{0}^{t} g(t-s)(\nabla u(t)-\nabla u(s)) \mathrm{d} s \mathrm{~d} x\right| \\
\leq & \left|\int_{\Omega}\left(m_{3}+\frac{b}{2 c_{0}} E(0)\right) \nabla u(t) \int_{0}^{t} g(t-s)(\nabla u(t)-\nabla u(s)) \mathrm{d} s \mathrm{~d} x\right| \\
\leq & \delta|\nabla u|^{2}+\frac{m_{3}-l}{4 \delta}\left(m_{3}+\frac{b}{2 c_{0}} E(0)\right)^{2}(g \diamond \nabla u)(t), \\
\leq \sigma^{2}( & \left.\left|\int_{\Omega}\left(\sigma u \nabla u_{\Omega} \mathrm{d} x\right)^{2} l\right| \nabla u\right|^{2}+\frac{1}{4 l} \int_{\Omega}\left(\int_{0}^{t} g(t-s)(\nabla u(t)-\nabla u(s)) \mathrm{d} s\right)^{2} \mathrm{~d} x \\
\leq & \mid \int_{\Omega}\left(\int _ { 0 } g \left(t-\frac{-\sigma}{2 c_{0}} E(0) E^{\prime}(t)+\frac{m_{3}-l}{4 \delta}(g \diamond \nabla u)(t),\right.\right. \\
\leq & \left.\mid m_{3}-l\right)^{2}|\nabla u|^{2}+\left(2 \delta+\frac{1}{4 \delta}\right)\left(m_{3}-l\right)(g \diamond \nabla u)(t),
\end{aligned}
$$

and

$$
\begin{aligned}
& \left|\int_{\Gamma_{1}} h\left(u_{t}\right) \int_{0}^{t} g(t-s)(u(t)-u(s)) \mathrm{d} s \mathrm{~d} \Gamma\right| \\
\leq & \frac{1}{2} \int_{\Gamma_{1}} h^{2}\left(u_{t}\right) \mathrm{d} \Gamma+\frac{\left(m_{3}-l\right) B_{*}^{2}}{2}(g \diamond \nabla u)(t) .
\end{aligned}
$$

As for the the fifth and sixth terms on the right-hand side of (4.32), utilizing Hölder's inequality, Young's inequality, (2.9), (2.13), (2.14) and (4.22), we obtain,

$$
\begin{gathered}
\left.\left|\int_{\Gamma_{1}}\right| u\right|^{k(x)-1} u \int_{0}^{t} g(t-s)(u(t)-u(s)) \mathrm{d} s \mathrm{~d} \Gamma \mid \\
\leq \delta \max \left(\|u\|_{2 k^{+}, \Gamma_{1}}^{2 k^{+}},\|u\|_{2 k^{-}, \Gamma_{1}}^{2 k^{-}}\right)+\frac{\left(m_{3}-l\right) B_{*}^{2}}{4 \delta}(g \diamond \nabla u)(t) \\
\leq \delta \max \left(B_{*}^{2 k^{+}}|\nabla u|^{2 k^{+}}, B_{*}^{2 k^{-}}|\nabla u|^{2 k^{-}}\right)+\frac{\left(m_{3}-l\right) B_{*}^{2}}{4 \delta}(g \diamond \nabla u)(t) \\
\leq \delta \max \left(B_{*}^{2 k^{+}}\left(\frac{E(0)}{2 l c_{0}}\right)^{k^{+}-1}, B_{*}^{2 k^{-}}\left(\frac{E(0)}{2 l c_{0}}\right)^{k^{-}-1}\right)|\nabla u|^{2}+\frac{\left(m_{3}-l\right) B_{*}^{2}}{4 \delta}(g \diamond \nabla u)(t)
\end{gathered}
$$


and

$$
\begin{gathered}
\left.\left|\int_{\Omega}\right| u\right|^{p(x)-1} u \int_{0}^{t} g(t-s)(u(t)-u(s)) \mathrm{d} s \mathrm{~d} x \mid \\
\leq \delta \max \left(B^{2 p^{+}}|\nabla u|^{2 p^{+}}, B^{2 p^{-}}|\nabla u|^{2 p^{-}}\right)+\frac{\left(m_{3}-l\right) B^{2}}{4 \delta}(g \diamond \nabla u)(t) \\
\leq \delta \max \left(B^{2 p^{+}}\left(\frac{E(0)}{2 l c_{0}}\right)^{p^{+}-1}, B^{2 p^{-}}\left(\frac{E(0)}{2 l c_{0}}\right)^{p^{-}-1}\right)|\nabla u|^{2}+\frac{\left(m_{3}-l\right) B^{2}}{4 \delta}(g \diamond \nabla u)(t) .
\end{gathered}
$$

Exploiting Hölder's inequality, Young's inequality and $\left(H_{1}\right)$ to estimate the seventh term, we have

$$
\begin{gathered}
\left.\left|\int_{\Omega} u_{t} \int_{0}^{t} g^{\prime}(t-s)(u(t)-u(s)) \mathrm{d} s \mathrm{~d} x-\left(\int_{0}^{t} g(s) \mathrm{d} s\right)\right| u_{t}\right|^{2} \mid \\
\leq \delta\left|u_{t}\right|^{2}-\frac{g(0) B^{2}}{4 \delta}\left(g^{\prime} \diamond \nabla u\right)(t) .
\end{gathered}
$$

Then, combining these estimates (4.33)-(4.39), (4.32) becomes

$$
\begin{aligned}
\Psi^{\prime}(t) \leq & -\left(\int_{0}^{t} g(s) \mathrm{d} s-\delta\right)\left|u_{t}\right|^{2}+c_{2} \delta|\nabla u|^{2}+c_{3}(g \diamond \nabla u)(t) \\
& -\frac{g(0) B^{2}}{4 \delta}\left(g^{\prime} \diamond \nabla u\right)(t)+\frac{1}{2} \int_{\Gamma_{1}} h^{2}\left(u_{t}\right) \mathrm{d} \Gamma-\frac{\sigma}{2 c_{0}} E(0) E^{\prime}(t),
\end{aligned}
$$

where

$$
\begin{aligned}
c_{2}= & 1+2\left(m_{3}-l\right)^{2}+\max \left(B_{*}^{2 k^{+}}\left(\frac{E(0)}{2 l c_{0}}\right)^{k^{+}-1}, B_{*}^{2 k^{-}}\left(\frac{E(0)}{2 l c_{0}}\right)^{k^{-}-1}\right) \\
& +\max \left(B^{2 p^{+}}\left(\frac{E(0)}{2 l c_{0}}\right)^{p^{+}-1}, B^{2 p^{-}}\left(\frac{E(0)}{2 l c_{0}}\right)^{p^{-}-1}\right),
\end{aligned}
$$

and

$$
c_{3}=\left(m_{3}-l\right)\left(\frac{1+\left(m_{3}+\frac{b E(0)}{2 l c_{0}}\right)^{2}}{4 \delta}+2 \delta+\frac{1}{4 l}+\frac{B_{*}^{2}}{2}+\frac{B^{2}+B_{*}^{2}}{4 \delta}\right) .
$$

Since $g$ is continuous and $g(0)>0$, then there exists $t_{0}>0$ such that,

$$
\int_{0}^{t} g(s) \mathrm{d} s \geq \int_{0}^{t_{0}} g(s) \mathrm{d} s=g_{0}, \forall t \geq t_{0} .
$$


Hence, we conclude from (4.23), (4.6), (4.31), and (4.40) that

$$
\begin{gathered}
G^{\prime}(t)=M E^{\prime}(t)+\varepsilon \Phi^{\prime}(t)+\Psi^{\prime}(t) \\
\leq-\left(\frac{M}{2}-\frac{g(0) B^{2}}{4 \delta}\right)\left(-\left(g^{\prime} \diamond \nabla u\right)(t)\right)-\left(g_{0}-\delta-\varepsilon\right)\left|u_{t}\right|^{2} \\
+\left(c_{2} \delta-\frac{\varepsilon l}{4}\right)|\nabla u|^{2}+\left(c_{3}+\frac{\left(m_{3}-l\right) \varepsilon}{2 l}\right)(g \diamond \nabla u)(t) \\
+\left(\frac{1}{2}+\frac{2 B_{*}^{2} \varepsilon}{l}\right) \int_{\Gamma_{1}} h^{2}\left(u_{t}\right) \mathrm{d} \Gamma-\frac{\sigma}{2 c_{0}} E(0) E^{\prime}(t) \\
+\varepsilon\left(\int_{\Omega}|u|^{p(x)+1} \mathrm{~d} x+\int_{\Gamma_{1}}|u|^{k(x)+1} \mathrm{~d} \Gamma\right) .
\end{gathered}
$$

At this point, we choose $\varepsilon>0$ small enough so that Lemma4.4 holds and $\varepsilon<\frac{g_{0}}{2}$. Once $\varepsilon$ is fixed, we choose $\delta$ to satisfy

$$
\delta<\min \left(\frac{g_{0}}{4}, \frac{\varepsilon l}{8 c_{2}}\right)
$$

and then pick $M$ sufficiently large such that $M>\frac{g(0) B^{2}}{2 \delta}$. Thus, for all $t \geq t_{0}$, we arrive at

$$
\begin{aligned}
G^{\prime}(t) \leq & -\frac{\varepsilon l}{8}|\nabla u|^{2}-\frac{g_{0}}{4}\left|u_{t}\right|^{2}+c_{4}(g \diamond \nabla u)(t)+c_{5} \int_{\Gamma_{1}} h^{2}\left(u_{t}\right) \mathrm{d} \Gamma \\
& -c_{6} E(0) E^{\prime}(t)+\varepsilon\left(\int_{\Omega}|u|^{p(x)+1} \mathrm{~d} x+\int_{\Gamma_{1}}|u|^{k(x)+1} \mathrm{~d} \Gamma\right) \\
\leq & -\frac{\varepsilon l}{4\left(m_{3}-g_{0}\right)} \frac{1}{2}\left(m_{3}-\int_{0}^{t} g(s) \mathrm{d} s\right)|\nabla u|^{2}-\frac{g_{0}}{4}\left|u_{t}\right|^{2} \\
& +c_{4}(g \diamond \nabla u)(t)+c_{5} \int_{\Gamma_{1}} h^{2}\left(u_{t}\right) \mathrm{d} \Gamma-c_{6} E(0) E^{\prime}(t) \\
& +\varepsilon\left(\int_{\Omega}|u|^{p(x)+1} \mathrm{~d} x+\int_{\Gamma_{1}}|u|^{k(x)+1} \mathrm{~d} \Gamma\right) .
\end{aligned}
$$

with some positive constants $c_{i}, i=4,5,6$. Additionally, observing the fact that $\frac{\varepsilon l}{4\left(m_{3}-g_{0}\right)}<g_{0}$ due to $\varepsilon<g_{0}$ and $\frac{l}{\left(m_{3}-g_{0}\right)}<1$ and employing the definition of $E(t)$ by (4.3) and using $|\nabla u|^{2} \leq \frac{E(0)}{2 l c_{0}}$ by (4.22), we deduce that

$$
\begin{gathered}
G^{\prime}(t) \leq-c_{7} E(t)+\frac{c_{7} b}{4}|\nabla u|^{4}+\left(c_{4}+\frac{c_{7}}{2}\right)(g \diamond \nabla u)(t) \\
+c_{5} \int_{\Gamma_{1}} h^{2}\left(u_{t}\right) \mathrm{d} \Gamma-c_{6} E(0) E^{\prime}(t)+\varepsilon c_{8}\left(\int_{\Omega}|u|^{p(x)+1} \mathrm{~d} x+\int_{\Gamma_{1}}|u|^{k(x)+1} \mathrm{~d} \Gamma\right) \\
\leq-\alpha_{1} E(t)+\left(c_{4}+\frac{c_{7}}{2}\right)(g \diamond \nabla u)(t)+c_{5} \int_{\Gamma_{1}} h^{2}\left(u_{t}\right) \mathrm{d} \Gamma-c_{6} E(0) E^{\prime}(t),
\end{gathered}
$$

where

$$
c_{7}=\frac{\varepsilon l}{4\left(m_{3}-g_{0}\right)},
$$




$$
c_{8}=\max \left(1-\frac{l}{4\left(p^{-}+1\right)\left(m_{3}-g_{0}\right)}, 1-\frac{l}{4\left(k^{-}+1\right)\left(m_{3}-g_{0}\right)}\right)>0
$$

and

$$
\alpha_{1}=c_{7}-\left(\frac{c_{7} b}{8 l^{2} c_{0}} E(0)+\varepsilon \frac{c_{8}}{2 l c_{0}}\left(\begin{array}{l}
\max \left(B^{p^{+}+1}\left(\frac{E(0)}{2 l c_{0}}\right)^{\frac{p^{+}-1}{2}}, B^{p^{-}+1}\left(\frac{E(0)}{2 l c_{0}}\right)^{\frac{p^{-}-1}{2}}\right), \\
\max \left(B_{*}^{k^{+}+1}\left(\frac{E(0)}{2 l c_{0}}\right)^{\frac{k^{+}-1}{2}}, B_{*}^{k^{-}+1}\left(\frac{E(0)}{2 l c_{0}}\right)^{\frac{k^{-}-1}{2}}\right)
\end{array}\right)\right) .
$$

Hence, if $E(0)$ is small enough, then not only the condition $E(0)<\mathrm{d}$ is satisfied, but also $\alpha_{1}>0$ is assured. Therefore, we have, for $t \geq t_{0}$,

$$
G^{\prime}(t) \leq-\alpha_{1} E(t)+\alpha_{2}(g \diamond \nabla u)(t)+\alpha_{3} \int_{\Gamma_{1}} h^{2}\left(u_{t}\right) \mathrm{d} \Gamma-\alpha_{4} E(0) E^{\prime}(t),
$$

where $\alpha_{i}, i=1, \ldots, 4$ are all positive constants. This completes the proof.

Before stating our main result, we need to recall that if $\varphi$ is a proper convex function from $\mathbb{R}$ to $\mathbb{R} \cup\{\infty\}$, then its convex conjugate $\varphi^{*}$ is defined as

$$
\varphi^{*}(y)=\sup _{x \in \mathbb{R}}\{x y-\varphi(x)\}
$$

Now, we are in a position to state our main result by adopting and modifying the arguments in $[18,39,20]$. We consider the following partition of $\Gamma_{1}$

$$
\Gamma_{1}^{+}=\left\{x \in \Gamma_{1}|| u_{t} \mid>r\right\}, \Gamma_{1}^{-}=\left\{x \in \Gamma_{1}|| u_{t} \mid \leq r\right\} .
$$

Theorem 4.6. Assume that the conditions of 4.5 are valid, then, for each $t_{0}>0$ and $k_{1}, k_{2}$ and $\varepsilon_{0}$ are positive constants, the solution energy of (1.1)-(1.4) satisfies

$$
E(t) \leq k_{2} H_{1}^{-1}\left(k_{1} \int_{0}^{t} \zeta(s) \mathrm{d} s\right), t \geq t_{0}
$$

where

$$
H_{1}(t)=\int_{t}^{1} \frac{1}{H_{2}(s)} \mathrm{d} s
$$

and

$$
H_{2}(t)=\left\{\begin{array}{c}
t, \text { if } H \text { is linear on }[0, r] \\
t H^{\prime}\left(\varepsilon_{0} t\right), \quad \text { if } H^{\prime}(0)=0 \text { and } H^{\prime \prime}>0 \text { on }(0, r] .
\end{array}\right.
$$

Proof. The global existence of solution $u$ of (1.1)-(1.4) is guaranteed directly by Theorem 4.3. Next, we consider the following two cases: $(i) H$ is linear on $[0, r]$ and $(i i)$ $H^{\prime}(0)=0$ and $H^{\prime \prime}>0$ on $(0, r]$.

Case 1. $H$ is linear on $[0, r]$. In this case, there exists $\alpha_{1}^{\prime}>0$ such that $|h(s)| \leq \alpha_{1}^{\prime}|s|$, for all $s \in \mathbb{R}$. By (4.6), we have

$$
\int_{\Gamma_{1}} h^{2}\left(u_{t}\right) \mathrm{d} \Gamma \leq \alpha_{1}^{\prime} \int_{\Gamma_{1}} u_{t} h\left(u_{t}\right) \mathrm{d} \Gamma \leq-\alpha_{1}^{\prime} E^{\prime}(t),
$$

which together with (4.42) implies that

$$
\left(G(t)+c_{9} E(t)\right)^{\prime} \leq-\alpha_{1} H_{2}(E(t))+\alpha_{2}(g \diamond \nabla u)(t)
$$


where $H_{2}(s)=s$ and $c_{9}=\alpha_{1}^{\prime} \alpha_{3}+\alpha_{4} E(0)$.

Case 2. $H^{\prime}(0)=0$ and $H^{\prime \prime}>0$ on $(0, r]$. In this case, we first estimate $\int_{\Gamma_{1}} h^{2}\left(u_{t}\right) \mathrm{d} \Gamma$ on the right-hand side of (4.42). Given (4.2), noting that $H^{-1}$ is concave and increasing and using the well-known Jensen's inequality and (4.6), we deduce that

$$
\begin{aligned}
\int_{\Gamma_{1}} h^{2}\left(u_{t}\right) \mathrm{d} \Gamma & =\int_{\Gamma_{1}} h^{2}\left(u_{t}\right) \mathrm{d} \Gamma+\int_{\Gamma_{1}} h^{2}\left(u_{t}\right) \mathrm{d} \Gamma \\
& \leq M_{1} \int_{\Gamma_{1}^{+}} u_{t} h\left(u_{t}\right) \mathrm{d} \Gamma+\int h_{\Gamma_{1}^{-}} h^{2}\left(u_{t}\right) \mathrm{d} \Gamma \\
& \leq-M_{1} E^{\prime}(t)+\int_{\Gamma_{1}^{-}} H^{-1}\left(u_{t} h\left(u_{t}\right)\right) \mathrm{d} \Gamma \\
& \leq-M_{1} E^{\prime}(t)+\frac{1}{c_{10}} H^{-1}\left(c_{10} \int_{\Gamma_{1}^{-}}\left(u_{t} h\left(u_{t}\right)\right) \mathrm{d} \Gamma\right) \\
& \leq-M_{1} E^{\prime}(t)+\frac{1}{c_{10}} H^{-1}\left(-c_{10} E^{\prime}(t)\right),
\end{aligned}
$$

where $c_{10}=\frac{1}{\left|\Gamma_{1}^{-}\right|}$. Hence, (4.42) becomes

$$
G_{1}^{\prime}(t) \leq-\alpha_{1} E(t)+\alpha_{3}\left|\Gamma_{1}^{-}\right| H^{-1}\left(-c_{10} E^{\prime}(t)\right)+\alpha_{2}(g \diamond \nabla u)(t), \forall t \geq t_{0},
$$

where

$$
G_{1}(t)=G(t)+\left(M_{1} \alpha_{3}+\alpha_{4} E(0)\right) E(t) .
$$

Now, we define

$$
G_{2}(t)=H^{\prime}\left(\varepsilon_{0} E(t)\right) G_{1}(t)+\beta E(t),
$$

where $\varepsilon_{0}>0$ and $\beta>0$ to be determined later. Then, using $E^{\prime}(t) \leq 0, H^{\prime \prime}(t) \geq 0$, and (4.48), we obtain

$$
\begin{gathered}
G_{2}^{\prime}(t)=\varepsilon_{0} E^{\prime}(t) H^{\prime \prime}\left(\varepsilon_{0} E(t)\right) G_{1}(t)+H^{\prime}\left(\varepsilon_{0} E(t)\right) G_{1}^{\prime}(t)+\beta E^{\prime}(t) \\
\leq-\alpha_{1} H^{\prime}\left(\varepsilon_{0} E(t)\right) E(t)+\alpha_{2} H^{\prime}\left(\varepsilon_{0} E(t)\right)(g \diamond \nabla u)(t) \\
+c_{11} H^{\prime}\left(\varepsilon_{0} E(t)\right) H^{-1}\left(-c_{10} E^{\prime}(t)\right)+\beta E^{\prime}(t) .
\end{gathered}
$$

To estimate the fourth term in the right hand side of (4.51), let $H^{*}$ be the conjugate function of the convex function $H$ defined by (4.43), then

$$
a b \leq H^{*}(a)+H(b) \text { for } a, b \geq 0 .
$$

Moreover, due to the argument given in [6], it holds that

$$
H^{*}(s)=s\left(H^{\prime}\right)^{-1}(s)-H\left(\left(H^{\prime}\right)^{-1}(s)\right) \text { for } s \geq 0 .
$$

Further, using (4.53) and noting that $H^{\prime}(0)=0,\left(H^{\prime}\right)^{-1}$ is increasing and $H$ is also increasing yield

$$
H^{*}(s) \leq s\left(H^{\prime}\right)^{-1}(s), s \geq 0
$$


Taking $H^{\prime}\left(\varepsilon_{0} E(t)\right)=a$ and $H^{-1}\left(-c_{10} E^{\prime}(t)\right)=b$ in (4.51), applying (4.54) and (4.52), and noting that $0 \leq H^{\prime}\left(\varepsilon_{0} E(t)\right) \leq H^{\prime}\left(\varepsilon_{0} E(0)\right)$ due to $H^{\prime}$ is increasing, to obtain

$$
\begin{gathered}
G_{2}^{\prime}(t) \leq-\alpha_{1} H^{\prime}\left(\varepsilon_{0} E(t)\right) E(t)+c_{11} H^{*}\left(H^{\prime}\left(\varepsilon_{0} E(t)\right)\right) \\
+c_{13}(g \diamond \nabla u)(t)+\left(\beta-c_{12}\right) E^{\prime}(t) \\
\leq-\left(\alpha_{1}-c_{11} \varepsilon_{0}\right) H^{\prime}\left(\varepsilon_{0} E(t)\right) E(t)+c_{13}(g \diamond \nabla u)(t)+\left(\beta-c_{12}\right) E^{\prime}(t)
\end{gathered}
$$

with $c_{12}=c_{10} c_{11}$ and $c_{13}=\alpha_{2} H^{\prime}\left(\varepsilon_{0} E(0)\right)>0$. Thus, choosing $0<c_{11} \varepsilon_{0}<\alpha_{1}$, $\beta>c_{12}$ and using $E^{\prime}(t) \leq 0$ by (4.6), we have

$G_{2}^{\prime}(t) \leq-c_{14} H^{\prime}\left(\varepsilon_{0} E(t)\right) E(t)+c_{13}(g \diamond \nabla u)(t)=-c_{14} H_{2}(E(t))+c_{13}(g \diamond \nabla u)(t)$,

where $H_{2}(s)=s H^{\prime}\left(\varepsilon_{0} s\right)$ and $c_{14}$ is a positive constant.

Let

$$
F_{1}(t)=\left\{\begin{array}{l}
G(t)+c_{9} E(t), \text { if } H \text { is linear on }[0, r], \\
G_{2}(t), \text { if } H^{\prime}(0)=0 \text { and } H^{\prime \prime}>0 \text { on }(0, r] .
\end{array}\right.
$$

Then, by Lemma 4.4 and the definition of $G_{2}$ by (4.49)-(4.50), there exist $\beta_{1}^{\prime}, \beta_{2}^{\prime}>0$ such that

$$
\beta_{2}^{\prime} E(t) \leq F_{1}(t) \leq \beta_{1}^{\prime} E(t),
$$

which is equivalent to $E(t)$, and from (4.47) and (4.55), we have

$$
F_{1}^{\prime}(t) \leq-c_{15} H_{2}(E(t))+c_{16}(g \diamond \nabla u)(t), t \geq t_{0},
$$

where $c_{15}$ and $c_{16}$ denote some positive constants. In addition, using (4.56) and $\xi(t) \leq$ $\xi(0)$ by $\left(H_{2}\right)$ and for $l_{1}=\beta_{1}^{\prime} \xi(0)+2 c_{16}>0$, we see that

$$
\xi(t) F_{1}(t)+2 c_{16} E(t) \leq l_{1} E(t), t \geq t_{0},
$$

Now, we define

$$
G_{3}(t)=\varepsilon_{1}\left[\xi(t) F_{1}(t)+2 c_{16} E(t)\right], 0<l_{1} \varepsilon_{1}<r,
$$

which is equivalent to $E(t)$ by (4.56). Thanks to (4.57), (2.10) and (4.6), we arrive at

$$
\begin{gathered}
G_{3}^{\prime}(t)=\varepsilon_{1}\left[\xi^{\prime}(t) F_{1}(t)+\xi(t) F_{1}^{\prime}(t)+2 c_{16} E^{\prime}(t)\right] \\
\leq-c_{15} \varepsilon_{1} H_{2}(E(t)) \xi(t)+c_{16} \varepsilon_{1} \xi(t)(g \diamond \nabla u)(t)+2 c_{16} \varepsilon_{1} E^{\prime}(t) \\
\leq-c_{15} \varepsilon_{1} H_{2}(E(t)) \xi(t)-c_{16} \varepsilon_{1}\left(g^{\prime} \diamond \nabla u\right)(t)+2 c_{16} \varepsilon_{1} E^{\prime}(t) \\
\leq-c_{15} \varepsilon_{1} H_{2}(E(t)) \xi(t) .
\end{gathered}
$$

Exploiting the fact that $H_{2}$ is increasing, using (4.58) and using the fact that $0<$ $l_{1} \varepsilon_{1}<r$ by (4.59), we obtain

$$
\begin{gathered}
G_{3}^{\prime}(t) \leq-c_{15} \varepsilon_{1} \xi(t) H_{2}\left(\frac{1}{l_{1}}\left(\xi(t) F_{1}(t)+2 c_{16} E(t)\right)\right) \\
\leq-c_{15} \varepsilon_{1} \xi(t) H_{2}\left(\varepsilon_{1}\left(\xi(t) F_{1}(t)+2 c_{16} E(t)\right)\right)=-c_{15} \varepsilon_{1} \xi(t) H_{2}\left(G_{3}(t)\right) .
\end{gathered}
$$

Using that $H_{1}^{\prime}(t) H_{2}(t)=-1($ see $(4.45))$, we see that

$$
G_{3}^{\prime}(t) H_{1}^{\prime}\left(G_{3}(t)\right) \geq c_{15} \varepsilon_{1} \xi(t), t \geq t_{0} .
$$


Integrating this over $\left(t_{0}, t\right)$ which implies, having in mind that $H_{1}^{-1}$ is decreasing on $(0, r]$, that

$$
\begin{aligned}
& G_{3}(t) \leq H_{1}^{-1}\left(H_{1}\right.\left.\left(G_{3}(0)\right)+c_{15} \varepsilon_{1} \int_{0}^{t} \xi(s) \mathrm{d} s-c_{15} \varepsilon_{1} \int_{0}^{t_{0}} \xi(s) \mathrm{d} s\right) \\
& \leq H_{1}^{-1}\left(c_{15} \varepsilon_{1} \int_{0}^{t} \xi(s) \mathrm{d} s\right)
\end{aligned}
$$

where we need $\varepsilon_{1}>0$ sufficiently small so that $H_{1}\left(G_{3}(0)\right)-c_{15} \varepsilon_{1} \int_{0}^{t_{0}} \xi(s) \mathrm{d} s>0$.

Consequently, from the equivalent relation of $G_{3}$ and $E$ yields

$$
E(t) \leq k_{2} H_{1}^{-1}\left(k_{1} \int_{0}^{t} \xi(s) \mathrm{d} s\right), t \geq t_{0},
$$

where $k_{1}$ and $k_{2}$ are positive constants. Hence, this completes the proof.

Remark 4.7. Because $\lim _{t \rightarrow 0} H_{1}(t)=\infty$ (see (4.46)), thus, if $\int_{0}^{\infty} \xi(s) \mathrm{d} s=\infty$, we get the stability of system (1.1)-(1.4), in the other words, $\lim _{t \rightarrow+\infty} E(t)=0$.

\section{References}

[1] Aboulaich, R., Meskine, D., Souissi, A., New diffusion models in image processing, Comput. Math. Appl, 56(2008), no. 4, 874-882.

[2] Antontsev, S.N., Shmarev, S.I., Elliptic Equations with Anisotropic Nonlinearity and Nonstandard Growth Conditions, a Handbook of Differential Equations, Stationary Partial Differential Equations, Elsevier/North Holland, Amsterdam, vol. 3, 2006.

[3] Antontsev, S.N., Shmarev, S.I., Blow-up of solutions to parabolic equations with nonstandard growth conditions, J. Comput. Appl. Math., 234(2010), no. 9, 2633-2645.

[4] Antontsev, S.N., Shmarev, S., Evolution PDES with Nonstandard Growth Conditions: Existence, Uniqueness, Localization, Blow-up, 1 ed., Atlantis Studies in Differential Equations, 4 Atlantis Press, 2015.

[5] Antontsev, S.N., Zhikov, V., Higher integrability for parabolic equations of $p(x, t)$ Laplacian type, Adv. Differential Equations, 10(2005), no. 9, 1053-1080.

[6] Arnold, V.I., Mathematical Methods of Classical Mechanics, Graduate Texts in Mathematics, vol. 10.1007/978-1-4757-2063-1, 1989.

[7] Chen, Y., Levine, S., Rao, M., Variable exponent, linear growth functionals in image restoration, SIAM J. Appl. Math, 66(2006), 1383-1406.

[8] Claudianor, O.A., Marcelo, M.C., On existence, uniform decay rates and blow up for solutions of the $2-d$ wave equation with exponential source, Calculus of Variations and Partial Differential Equations, 34(2009), 377-411.

[9] David, R.P., Rammaha, M.A., Global existence and non-existence theorems for nonlinear wave equations, Indiana University Mathematics Journal, 51(2002).

[10] Diening, L., Hästo, P., Harjulehto, P., Ruzicka, M., Lebesgue and Sobolev Spaces with Variable Exponents, Springer Lecture Notes, Springer-Verlag, vol. 2017, Berlin, 2011.

[11] Diening, L., Ruzicka, M., Calderon Zygmund operators on generalized Lebesgue spaces $L^{p(x)}(\Omega)$ and problems related to fluid dynamics, Preprint Mathematische Fakultät, Albert-Ludwigs-Universität Freiburg, 120(2002), 197-220. 
[12] Edmunds, D.E., Rákosník, J., Sobolev embeddings with variable exponent, Studia Math., 143(2000), no. 2, 424-446.

[13] Fan, X.L., Boundary trace embedding theorems for variable exponent Sobolev spaces, J. Math. Anal. Appl, 339 (2008), no. 2, 1395-1412.

[14] Fan, X., Shen, G., Multiplicity of positive solutions for a class of inhomogeneous Neumann problems involving the $p(x)$-Laplacian, Nonlinear Differ. Equ. Appl., 16(2009), $255-271$.

[15] Fan, X., Shen, G., Zhao, D., Sobolev embedding theorems for spaces $W^{k, p(x)}(\Omega)$, J. Math. Anal. Appl, 262 (2001), 749-760.

[16] Fan, X.L., Zhao, D., On the space $L^{p(x)}(\Omega)$ and $W^{m, p(x)}(\Omega)$, J. Math. Anal. Appl., 263(2001), 424-446.

[17] $\mathrm{Fu}, \mathrm{Y} .$, The existence of solutions for elliptic systems with nonuniform growth, Studia Math., 151(2002), 227-246.

[18] Guessmia, A., Messaoudi, S.A., General energy decay estimates of Timoshenko systems with frictional versus viscoelastic damping, Mathematical Methods in the Applied Sciences, 32(2009), 2102-2122.

[19] Guesmia, A., Messaoudi, S.A., Webler, C.M., Well-posedness and optimal decay rates for the viscoelastic Kirchhoff equation, Boletim da Sociedade Paranaense de Matemática, 35(2017), 203-224.

[20] Ha, T.G., On viscoelastic wave equation with nonlinear boundary damping and source term, Commun Pure Appl Anal., 6(2010), 1543-1576.

[21] Howard, A.L., Grozdena, T., Blow up of solutions of the Cauchy problem for a wave equation with nonlinear damping and source terms and positive initial energy, Proceedings of the American Mathematical Society, 129(2001), 341-361.

[22] Howard, A.L., Jame, S., Global nonexistence theorems for quasilinear evolution equations with dissipation, Archive for Rational Mechanics and Analysis, 137(1997), 341-361.

[23] Kovàcik, O., Rákosnik, J., On spaces $L^{p(x)}(\Omega)$ and $W^{1, p(x)}(\Omega)$, Czechoslovak Math. J., 41(1991).

[24] Levine, H.A., Instability and nonexistence of global solutions of nonlinear wave equation of the form $p u_{t t}=a u+f(u)$, Trans. Amer. Math. Sci., 192(1974), 1-21.

[25] Levine, H.A., Some additional remarks on the nonexistence of global solutions of nonlinear wave equation, SIAM J. Math. Anal, 5(1974), 138-146.

[26] Lian, S., Gao, W., Cao, C., Yuan, H., Study of the solutions to a model porous medium equation with variable exponent of nonlinearity, J. Math. Anal. Appl., 342(2008), no. 1, 27-38.

[27] Lions, J.L., Quelques méthodes de résolution des problèmes aux limites non linéaires, Dunod, Paris, 1966.

[28] Marcelo, M.C., Valéria, N.D.C., Irena, L., Well-posedness and optimal decay rates for the wave equation with nonlinear boundary damping-source interaction, Journal of Differential Equations, 236(2007), 407-459.

[29] Miranda, M.M., Medeiros, L.A., Hidden regularity for semilinear hyperbolic partial differential equations, Ann. Fac. Sci. Toulouse Math., 1(1988), 103-120.

[30] Munoz, J.E.R., Global solution on a quasilinear wave equation with memory, Nonlinear Analysis: Theory, Methods, and Applications, 8B(1994), 289-303. 
[31] Ono, K., Global existence, decay, and blow-up of solutions for some mildly degenerate nonlinear Kirchhoff strings, J. Differential Equations, 137(1997), 273-301.

[32] Ono, K., On global solutions and blow-up solutions of nonlinear kirchoff string with nonlinear dissipations, J. Math. Anal. Appl., 216(1997), 321-342.

[33] Patrizia, P., James, S., Global nonexistence for abstract evolution equations with positive initial energy, Journal of Differential Equations, 150(1998), 203-214.

[34] Rahmoune, A., On the existence, uniqueness and stability of solutions for semilinear generalized elasticity equation with general damping, Acta Mathematica Sinica, English Series, 33(2017), no. 11, 1549-1564.

[35] Rahmoune, A., Semilinear hyperbolic boundary value problem associated to the nonlinear generalized viscoelastic equations, Acta Mathematica Vietnamica, 43(2018), no. 2, 219238.

[36] Rahmoune, A., Existence and asymptotic stability for the semilinear wave equation with variable-exponent nonlinearities, Journal of Mathematical Physics, 60(2019), 122701.

[37] Ricardo, T., Jiongmin, Y., On a quasilinear wave equation with memory, Nonlinear Analysis: Theory, Methods, and Applications, 16(1991), 61-78.

[38] Robert, T.G., Blow-up theorems for nonlinear wave equations, Mathematische Zeitschrift, 132(1973), 183-203.

[39] Shun-Tang, W., General decay and blow-up of solutions for a viscoelastic equation with nonlinear boundary damping-source interactions, Zeitschrift für Angewandte Mathematik und Physik (ZAMP), 63(2012), 65-106.

[40] Takeshi, T., Existence and asymptotic behavior of solutions to weakly damped wave equations of Kirchhoff type with nonlinear damping and source terms, Journal of Mathematical Analysis and Applications, 361(2010), 566-578.

[41] Tatar, N., Zaraï, A., Exponential stability and blow up for a problem with balakrishnantaylor damping, Demonstratio Mathematica, 44(2011), 67-90.

[42] Todorova, G., Vitillaro, E., Blow-up for nonlinear dissipative wave equations in $\mathbb{R}^{n}$, J. Math. Anal. Appl, 303(2005), 242-257.

Abita Rahmoune

Laboratory of Pure and Applied Mathematics,

Amar Telidji University-Laghouat 03000, Algeria

e-mail: abitarahmoune@yahoo.fr

Benyattou Benabderrahmane

Laboratory of Pure and Applied Mathematics,

Mohamed Boudiaf University-M'Sila 28000, Algeria

e-mail: bbenyattou@yahoo.com 